\title{
Theoriekonstruktion durch Theorienvergleich - eine soziologische Theorietechnik
}

\author{
Fabian Anicker
}

Eingegangen: 29. Oktober 2019 / Angenommen: 30. Oktober 2020 / Online publiziert: 10. Dezember 2020

(C) Der/die Autor(en) 2020

Zusammenfassung Theorienvergleiche sind nicht nur ein kollektives Unternehmen zur Sicherung fachlicher Standards. Sie können auch dem eigenen produktiven Umgang mit Theorienvielfalt dienen und eignen sich sogar für die Konstruktion neuer Theorien. Es wird gezeigt, dass in der Praxis soziologischer Theorie bereits eine Technik der Theoriekonstruktion durch Theorienvergleich besteht und diese implizite Technik in einem allgemeinen Schema expliziert werden kann. Die Strategie bei der Herausarbeitung dieser Theorietechnik besteht im rekonstruktiven Reverse Engineering von Schlüsseltexten der soziologischen Theorie. Dabei wird nicht nach den Inhalten, sondern nach den Texten zugrundeliegenden Strategien der Theoriekonstruktion und -darstellung gefragt und nach Möglichkeiten gesucht, diese in eine kontextunabhängige Form zu bringen. Durch diesen Beitrag zum methodischen Selbstbewusstsein der soziologischen Theorie soll die Praxis der Theoriekonstruktion leichter erlernbar und die Darstellung theoretischer Innovationen transparenter gemacht werden.

Schlüsselwörter Theorievergleich · Theorizing $\cdot$ Methode $\cdot$ Sozialwissenschaften Soziologie

F. Anicker $(\bowtie)$

Institut für Soziologie, Westfälische Wilhelms-Universität Münster

Scharnhorstraße 121, 48151 Münster, Deutschland

E-Mail: fabian.anicker@gmail.com 


\section{Theory Construction by Theory-Comparison-a Sociological Theorizing Technique}

Abstract Comparing sociological theories does not only serve the purpose of collectively defining disciplinary standards, but it is also suitable for dealing with the multiplicity of theories in a productive fashion and even for constructing new theories. This article aims to show that in the practice of sociological theory there already exists a technique of constructing theories by theory comparison and that this tacit technique can be made explicit in a general scheme. The strategy for carving out this theorizing technique is to reconstructively reverse engineer key texts in sociological theory. The analysis will not concentrate on the contents of the texts but rather on underlying strategies of theory construction and presentation and will aim to express these in a context-independent form. This contribution to the methodological self-consciousness of sociological theory is designed to render the practice of theory construction more accessible and make claims to theoretical innovation more transparent.

Keywords Method - Theorizing - Social science $\cdot$ Theory construction sociology

\section{Einleitung}

Der Kollektivsingular soziologischer Theorie präsentiert sich bei näherer Betrachtung als ein unübersichtliches Nebeneinander verschiedener Paradigmen und Ansätze. Ordnungsbildend wirken weder eine Matrix geteilter fachlicher Kernprobleme noch ein allgemeines System abstrakter theoretischer Grundlagen. Dominantes Sortierschema im Fachdiskurs ist vielmehr die Zurechnung miteinander verbundener Aussagen und theoretischer Festlegungen auf einzelne Ansätze, Theorieprogramme und stilbildende Autoren. Auch gegenüber Versuchen, durch Theorieintegration oder Anspruch auf Universalität der theoretischen Überlegungen aus diesem Partikularismus auszusteigen (etwa Luhmann 1984; Esser 1993), hat der Fachdiskurs bisher stets zuverlässig mit der Zuweisung einer Parzelle in der unvermindert pluralistischen Theorielandschaft reagiert: Nun gibt es eben auch Luhmannianerinnen und Esser-Schüler.

Strittig ist die Bewertung dieses Umstands. Die über die Pluralität der Ansätze ermöglichte Perspektivenvielfalt ist häufig als gegenstandsangemessene Stärke des Fachs hervorgehoben worden (Balog und Schülein 2008; Baumann und Bonacker 2008; Bonacker et al. 2008; Kneer und Schroer 2009; Burzan 2019). In der deutschen Theorienvergleichsdebatte herrschte hingegen eher der Grundimpuls vor, durch die Suche nach Grundverhältnissen zwischen (Greshoff et al. 2007; Greshoff und Schimank 2006; Lindemann 2006) oder allgemeinen Bezugsproblemen über den Theorien auf die sachliche Integration der Theorielandschaft zu dringen (Hondrich 1976; Schneider 1999; Anicker 2017) oder aber Rahmenbedingungen der Theoriekonkurrenz zu etablieren, die das Theorieangebot durch Falsifikation verringern (Opp und Wippler 1990; Haller 1999; Schmid 2004, 2009). Auch diese Versuche haben jedoch keineswegs zu einer Reduktion theoretischer Vielfalt beigetragen, sodass 
der Theoriepluralismus, ganz gleich ob man ihn begrüßt oder ablehnt, ein Faktum ist, mit dem alle Theoriediskussion in der Soziologie beginnen muss.

Die Tatsache des theoretischen Pluralismus ist allerdings nicht nur wissenschaftstheoretisch bezüglich der Kohärenz des Fachdiskurses von Belang. Auch aus der Perspektive derjenigen, die eigenständige Beiträge zur soziologischen Theorie liefern wollen, ist die Frage des Umgangs mit Theorienvielfalt virulent. Interessant ist in diesem Zusammenhang die Frage nach dem Zusammenhang von Kompetenzerwerb (Theoriedidaktik) und tatsächlicher Praxis der Theorie.

In der akademischen Lehre wird der Theorienpluralismus typischerweise über ein Nacheinander verschiedener ,klassischer“ Ansätze abgebildet: Man liest Weber, Marx, Bourdieu, Luhmann und reitet darüber hinaus die persönlichen Steckenpferde der jeweiligen Lehrstuhlinhaber (vgl. Lenger et al. 2014). Dieses Vorgehen hat durchaus Vorteile. Viele der Problemstellungen soziologischer Klassiker sind von anhaltendem Interesse und ihre Grundlegungen haben die Fachentwicklung stark geprägt. Andererseits kann der Wissensstand der soziologischen Theorie auf diese Weise nicht besonders stark konzeptionell integriert werden; er besteht in einer Reihung je unabhängiger Theorieangebote, die zwar rekombiniert, nicht aber in einen übergreifenden theoretischen Rahmen gebracht werden können. Es bleibt den Studierenden weitgehend selbst überlassen, wie sie sich zu der Theorievielfalt verhalten und ob sie von der Sammlung partikularer Positionen abstrahieren oder nicht.

Dies ändert sich jedoch, sobald eigenständige Beiträge zur Theoriediskussion angestrebt werden. Wenn die Perspektivenvielfalt der Soziologie für die theoretische Gegenstandserschließung tatsächlich produktiv wirken soll, müssen inhaltliche Brückenschläge zwischen bestehenden theoretischen Perspektiven und eigener Theorieleistung erreicht werden.

Dafür reicht es nicht aus, verschiedene theoretische Begriffslogiken zu beherrschen und anwenden zu können. Man muss auch verstehen, an welchen Punkten man mit gegebenen Theorien nicht mehr weiterkommt und wo man ansetzen müsste, um den möglicherweise zu engen Rahmen theoretischer Vorannahmen zu transzendieren. Zudem muss der Anspruch, nicht bloß eine neue, sondern auch eine in spezifischer Hinsicht sachlich überlegene theoretische Leistung vollbracht zu haben, in einem Diskurs vertreten werden, der mit den soziologischen Theorien auch theoretische Gütekriterien weitgehend partikularisiert hat. In der Theoriearbeit muss nicht nur die Theorie, sondern auch ihr intendierter „Rechtfertigungskontext“" (Reichenbach 1983, S. 3) mitkonstruiert werden. Konstruktive Theoriearbeit erfordert deshalb synthetisierende Abstraktionsleistungen, die geteilte Bezugspunkte zwischen bestehenden Theorien sichtbar machen und die eigene Theorie in diesem Kontext verorten. Die bloße Rekonstruktion von Klassikern und ihrer Grundannahmen bereitet auf diese Aufgabe nicht vor.

Man kann daher vermuten, dass der didaktische Gehalt der Theorieausbildung und das Anforderungsprofil praktischer Theoriearbeit auseinanderfallen (z. B. Swedberg 2012). Insbesondere scheint es wahrscheinlich, dass eine auf Theoriefamilien und -ansätze fokussierte Theorieausbildung dazu tendiert, allgemeine Strategien, Methoden und Techniken der Theoriekonstruktion zu verdecken, die für die praktische Theoriearbeit wertvolles „Handwerkszeug“ darstellen könnten. In diesem Artikel soll daher der Versuch gemacht werden, eine solche, über bestimmte theoreti- 
sche Präferenzen hinausreichende Technik der Theoriekonstruktion zu bestimmen: die Theoriekonstruktion durch Theorienvergleich. Damit schließen wir der Intention nach, nicht jedoch in vielen Grundannahmen zur theoretischen Praxis, an das sogenannte Theorizing Movement an (Swedberg 2017), in dem es um die Suche nach Möglichkeiten geht, den Erwerb produktiver soziologischer Theoriekompetenz zu erleichtern. Der folgende Abschnitt verortet unser methodisches Unternehmen in dieser Strömung, plädiert aber für einen inklusiveren und für genuine Theoriearbeit offenen Begriff des Theorizing. Der Hauptteil des Artikels ist dem Versuch gewidmet, eine bestimmte Technik der theorienvergleichenden Theoriekonstruktion zu explizieren und somit konkret zum methodischen Selbstbewusstsein soziologischer Theorie beizutragen. In Abschn. 3 wird die Grundstrategie der Untersuchung vorgestellt. Abschnitt 4 erläutert den für konstruktive und kritische Theorienvergleiche zentralen Grundsatz, dass Theorien als Lösungen von Problemen zu verstehen sind. Herzstück der Untersuchung sind drei Studien zur Praxis der Theoriekonstruktion von Bourdieu, Habermas und Luhmann (Abschn. 5), in denen gezeigt wird, dass die drei Autoren das Schema von Problem und Lösung explizit für die kritische Rekonstruktion von Vorgängertheorien verwenden und sie Grundfragen und Ausgangspunkte ihrer eigenen Theorie durch kritisch-vergleichenden Umgang mit der theoretischen Tradition erarbeiten. Wegen der weitreichenden theorietechnischen Konvergenz kann im letzten Abschnitt (Abschn. 6) eine allgemeine, von partikularen Ansätzen unabhängige Technik der Theoriekonstruktion durch Theorienvergleich angegeben werden.

\section{Theorizing}

Unter dem Schlagwort Theorizing laufen derzeit Versuche, theoretisches Know-how zu explizieren und dadurch die Zugangshürden zur aktiven Teilnahme am Theoriediskurs zu senken (Weick 1989; Abbott 2004; Swedberg 2012, 2014a; Farzin und Laux 2014; Tavory 2016).

Aufgedeckt werden Muster, Motive und Strategien der Theoriebildung, die sowohl forschungspraktischen Nutzen haben als auch gezielt gelehrt werden können. Bisherige Veröffentlichungen $\mathrm{zu}$ diesem Thema zielen vor allem auf forschungshandwerkliche Strategien und Kunstgriffe der Theoriekonstruktion im ,context of discovery" ab (Abbott 2004; Swedberg 2014b).

Bei ähnlicher Stoßrichtung bestehen jedoch Divergenzen zum hier verfolgten Ziel. In der Theorizing-Literatur geht es vor allem um phänomen- oder datenbezogene Theoriebildung mit Originalitätsanspruch, nicht um anspruchsvollere Theorieformen mit Allgemeinheitsanspruch. Insbesondere wird fast allgemein die Auffassung vertreten, dass neue Theorieentwicklungen weitgehend unabhängig vom bestehenden soziologischen Theoriediskurs und anderen Theorien erfolgen sollten.

Bei Swedberg etwa stehen nicht Theorien und ihre Probleme am Beginn des Theorizing, sondern Beobachtungen, Daten und Auffälligkeiten. In einem Bottomup-Prozess des abduktiven Schließens sollen aus Beobachtungen schrittweise Konzepte, Zusammenhänge und schließlich Erklärungen abgeleitet werden (Swedberg 2014a, S. 29-124). Die Erklärung bezeichnet dabei nicht den Bezug von Theorie 
und Daten (vgl. Popper 1935, S. 26 ff.), sondern gilt als Perfektionsform der Theorie selbst: Alle Theorie soll Erklärung werden (vgl. Krause 2016; zur Vielfalt von Theorietypen s. hingegen Abend 2008). Mit dieser Aufwertung der vermeintlich vortheoretischen „Daten“ als Ausgangspunkt und Zielkontext der Theoriebildung geht eine Abwertung bestehender Theorien und klassischer „Theoriearbeit“ einher. Theorizing bedeute eine Präferenz für die Entwicklung eigener Ideen, statt sich an den Theorien ,anderer Leute“ zu bedienen (Swedberg 2014a, S. 215); es komme auf die Ausschöpfung der eigenen Ressourcen an (Hammond 2018, S. 3). Theorien, die ,aus selbstreferentiellen Lektüren, Vergleichen oder Erweiterungen bestehender Theorien generiert werden“ seien ,defizitär, da sie auf den Einbau unmittelbarer und konkreter Wirklichkeitsbezüge verzichten und stattdessen auf kommunikative Anschlussfähigkeit an den soziologischen Diskurs abzielen ..." (Farzin und Laux 2016, S. 243). ${ }^{1}$ Führende Vertreter der Strömung verkünden mit ähnlichem Impetus, aber größerer Konsequenz, andere soziologische Theorie gar nicht mehr zu lesen es lohne sich nicht (Abbott 2010). Die häufig begründungsfrei vorgetragene Ablehnung sowohl von intertheoretischer Auseinandersetzung als auch von ,abstract theory“ oder ,grand theory“ und allem, was sich allzu weit vom möglichst konkret angesetzten ,puzzle“ einer Forschungssituation entfernt, steht gewissermaßen vor der Klammer. Bestehende soziologische Theorie wird dann im Grenzfall nicht als Anregung für die eigene Theoriearbeit, sondern als Hindernis für die Entfaltung der eigenen Kreativität wahrgenommen (s. Anicker 2019).

Es steht zu vermuten, dass die Blindheit der Theorizing-Strömung für die Rolle des bestehenden Theoriediskurses in der Theoriekonstruktion auf problematischen wissenschaftstheoretischen Auffassungen ruht (so auch Carleheden 2019). Insbesondere sind die empiristische Interpretation von Theorien als Schlüsse aus empirischen Beobachtungen und die Entgegensetzung von Systematizität und Innovativität in der gängigen Theorizing-Literatur zu hinterfragen.

Statt Beobachtungen als exklusive Auslöser theoretischer Kreativität zu fassen, wäre anzuerkennen, dass viele der interessanten Beobachtungen von Soziologen erst dadurch ermöglicht werden, dass sie im Laufe ihrer Fachsozialisation und natürlich durch die Lektüre von anderen Theorien gelernt haben, die Dinge mit „soziologischen Augen“ (Collins 1998) zu betrachten. Die Alternative zu theoretischer Vorbildung ist nicht Originalität, sondern die „Spontansoziologie“ von Laien (vgl. Bourdieu et al. 2011). Ferner herrscht in Wissenschaftssoziologie und -theorie weitgehend Konsens darüber, dass sich theoretisch und empirisch folgenreiche Paradigmenwechsel nicht durch vermeintlich unvoreingenommene Analysen von Daten vollziehen. Vielmehr bereitet erst der Nachweis von Defiziten bestehender Theorieperspektiven - die Ansammlung von „Anomalien“ (Kuhn) in Verbindung

\footnotetext{
1 Diese Behauptung versäumt es, die für das Theorizing sonst so relevanten Unterscheidungen zwischen Theoriekonstruktion und Theorieprüfung; Entdeckungs- und Rechtfertigungszusammenhang konsequent durchzuhalten. Ob Theorien empirisch gehaltvoll sind, entscheidet sich nicht an den Umständen der Theoriekonstruktion (also ob Theorien im Dialog mit anderen Theorien oder an empirischen Daten entwickelt wurden), sondern daran, ob sie für die Forschung hinreichend spezifische Vorgaben machen und Anschluss an sozialwissenschaftliche Methoden finden können. Nicht ob Theorien im beständigen Kontakt mit den empirischen Phänomenen entwickelt wurden, sondern ob sie eine Anleitung für den forschungspraktischen Umgang mit ihnen liefern, ist entscheidend (vgl. Carleheden 2016).
} 
mit möglichen alternativen Interpretationen - den Boden für neue Theorien (Fleck 1980; Kuhn 1970; Lakatos 1982; Toulmin 1983). Deshalb ist die Reflexion der Unzulänglichkeiten bestehender Theorien eine der wichtigsten Anregungsquellen für theoretische Innovationen.

Wenn es also richtig ist, Theoriekonstruktion nicht als Schöpfungsakt, sondern als einen Prozess der kritisch-konstruktiven Auseinandersetzung mit dem Bestehenden zu konzipieren, gibt es keine überzeugenden Gründe dafür, das Partikulare dem Allgemeinen, die datenbezogene Erklärung der Arbeit an theoretischen Grundbegriffen und das eigene dem fremden Gedankengut pauschal vorzuziehen. Die Auseinandersetzung mit bestehender Theorie ist kein unkreativer, rein reproduktiver Prozess. Im Gegenteil ist die kritisch-systematische Lektüre anderer Theorien für innovatives Denken äußerst anregend und für die Erreichung eines gewissen Problemniveaus sogar notwendig. Erst wenn die Beschränkung des Theorizing-Diskurses auf einen bestimmten Theorie- und Forschungstyp wegfällt, können auch jene Strategien und Techniken der Theoriebildung analysiert werden, die anspruchsvollen theoretischen Unternehmungen zugrunde liegen. Auch in der Manier der vielgescholtenen ,,armchair sociology" können neue theoretische Einsichten gewonnen werden. Tradition und Innovation sind keine Gegensätze, denn ohne die Kenntnis bestehender Theorie kommt man über mit exotischen Vokabeln aufgepeppte Alltagsansichten wohl kaum hinaus - oder wie es einer der Helden der ,armchair sociology“ formulierte:

... he who would arrive at important empirical conclusions transcending common sense cannot afford to neglect considerations of systematic theory (Parsons 1968, S. 12).

\section{Methodisches Vorgehen}

Die Behauptung, dass theoretische Innovationen auf theorienvergleichendem Wege möglich und für anspruchsvollere theoretische Entwürfe vielleicht sogar typisch sind, ist im Folgenden zu belegen. Der Beleg wird jedoch nicht in Form einer Abhandlung zum Wesen der Theorie erbracht, sondern besteht darin, einen bestimmten (sehr kleinen) Teil jenes impliziten Wissens zu explizieren, das komplexere theoretische Leistungen möglich macht.

Unsere ebenso einfache wie bisher kaum beachtete Frage lautet: Wie ,theoretisieren" Vertreter anspruchsvoller, über die Generalisierung phänomenbezogener Erklärungen hinausgehender Theorieformen? Welche impliziten Techniken und Strategien soziologischer Theoriekonstruktion lassen sich angeben? Unter „Techniken“ der Theoriekonstruktion werden Verfahrensweisen verstanden, die von ihren Verwendungskontexten (z. B. den Argumentationszusammenhängen konkreter Theorieentwürfe) abgelöst werden können. Gerade weil (Theorie-)Techniken simplifizieren und den Verweisungsreichtum von Theorien einschränken, können sie für die Praxis der soziologischen Theorie entlastend wirken (vgl. Luhmann 1990, S. $711 \mathrm{ff.}$., 2005a, S. 318). Unter „Strategie“ soll der kluge Mitteleinsatz zur Förderung eines gegebenen Zwecks (in unserem Fall: der Entwicklung und Präsentation theoretischer Entwürfe) verstanden werden. Diese Mittel können Techniken sein, aber die Praxis 
soziologischer Theorie erfordert auch eine Vielzahl von strategischen Entscheidungen, die nicht auf eine technische Basis gestellt werden können.

Das methodische Vorgehen der Untersuchung ist rekonstruktiv. Texte von sehr unterschiedlichen, aber in ihrem Anspruch auf grundlagentheoretische Innovation vergleichbaren Autoren werden auf ihre theorietechnische Konvergenz hin untersucht: Bourdieu, Habermas und Luhmann. Anzusetzen ist dabei nicht primär bei den Inhalten, sondern eher bei der Form und der Konstruktionsweise theoretischer Texte. Wir behaupten, dass sich trotz aller Unterschiede und polemisch unterstützter Lagerbildung eine ähnliche Technik der Theoriekonstruktion und Strategie der Theorieinszenierung in den Texten nachweisen lässt: die Theoriekonstruktion durch Theorienvergleich. Es ist zu zeigen, dass die theoriekonstruktiven Strategien von Autoren, die ganz unterschiedliche Pole in der soziologischen Theorie besetzen, mit demselben Vokabular artikuliert und auf dasselbe technische Grundprinzip zurückgeführt werden können. Der Sinn des Nachweises einer solchen theorietechnischen Übereinstimmung ist es, das methodische Selbstbewusstsein der soziologischen Theorie zu fördern: Das, was man sonst nur tun kann (,know how“), soll sagbar werden (,,know that"). Zunächst stellt sich allerdings die Vorfrage nach der Sprache, in der diese Konvergenz ausgedrückt werden soll.

\section{Das Vokabular von Problem und Lösung}

Nicht jede Art, soziologische Theorien zu lesen und $\mathrm{zu}$ vergleichen, ist für die Theoriekonstruktion produktiv. Es bedarf einer kritisch-systematischen Lektüre, die Theorie weder als Selbstzweck feiert noch als Weltanschauung oder Ausdruck der Meinungen ihrer Urheber behandelt. Für die Anleitung einer solchen Lektüre existiert in der soziologischen Theorie ein überraschend weitgehend geteiltes Schema. Sowohl in der Praxis des Theorienvergleichs als auch der Theoriekonstruktion lässt sich dieselbe Theorietechnik des Verstehens, d.h. der gedanklichen Organisation von theoretischen Inhalten, auffinden: das objektive Theorieverstehen über die Auslegung von Theorien auf Probleme. ${ }^{2}$ Theorien erschließen sich in ihrem objektiven Sinn als Antworten auf Fragen oder als Lösungen von Problemen und sind daher nicht an die Intentionen ihres Autors gebunden. Personennamen sind in der sachorientierten Theoriearbeit nur Kürzel für ein Bündel von Problemstellungen und theoretischen Grundentscheidungen. In diesem Punkt besteht eine Konvergenz zwischen hermeneutischen (Gadamer 1975), funktional-analytischen (Luhmann 2005a, 2005b, 2005c) und wissenschaftstheoretischen Ansätzen (Popper 1952, 1979; Toulmin 1983; für die Entfaltung dieser Konvergenzthese siehe Schneider 1991). Dabei ist das Frage/Antwort-Schema so grundlegend, dass Gadamer es für die Explikation der Struktur des Verstehens schlechthin nutzen kann (vgl. Gadamer 1975, S. 352 ff.).

\footnotetext{
2 Im Gegensatz zu Theorien, die alles Verstehen auf das Verstehen der Intentionen eines Sinnproduzenten beziehen, wird vorausgesetzt, dass der Sinn eines Textes gegenüber den Intentionen seiner Autorin eine intersubjektive und in diesem Sinne objektive Bedeutung hat. Man kann also z. B. die Systemtheorie verstehen, ohne dauernd bei Luhmann nachzufragen, und es ist auch möglich, objektiv richtige Implikationen der Theorie zu entdecken, an die Luhmann selbst nie gedacht hat.
} 
Es macht allerdings einen bedeutenden Unterschied, ob man das Schema von Frage und Antwort oder Problem und Lösung ${ }^{3}$ bloß stillschweigend verwendet oder ob man es bewusst nutzt, um Theorien zu interpretieren und ihre Auslegung zu fokussieren. Sprache steuert Aufmerksamkeit (Tomasello 2006) und ein explizites Problem/ Lösungs-Schema ist besonders geeignet, um unsere Aufmerksamkeit im Umgang mit Theorien zu steuern. In der Theorienvergleichsdebatte hat sich daher gerade für Versuche, Theorien sinnhaft in Bezug zu setzen, das Vokabular von Problem und Lösung als besonders nützlich erwiesen (Anicker 2017; Schneider 1999, 2008). Einer der Gründe dafür ist ganz einfach: Man behält leichter den Überblick. Die sprachliche Form erlaubt, in relativ bündiger Form auszudrücken, welchen Beitrag bestimmte Ideen für ein bestimmtes Ziel leisten. Formulieren wir versuchsweise das bisher Gesagte im Schema Problem/Lösung. Wir behaupten, dass es für das Problem, wie man die Inhalte wissenschaftlicher Theorien und anderer Texte am besten verstehen sollte, eine weitverbreitete und bewährte Lösung gibt - nämlich das Verständnis am Problem/Lösungs-Schema zu orientieren.

Der spezifisch theorienvergleichende Nutzen der Terminologie von Problem und Lösung liegt in der Möglichkeit der Gewinnung von Bezugspunkten zwischen Theorien durch die Erschließung abstrakter Bezugsprobleme. Theoretische Probleme werden erst durch die Interpretation von Theorien zugänglich und sind diesen insofern intern zugehörig, andererseits aber abstrahiert das Problem vom theoretischen Inhalt und schafft dadurch Raum für Alternativen. In den Worten des bereits erwähnten und in der Sache maßgeblichen Lehr- und Lehnstuhlinhabers:

Theory not only formulates what we know but also tells us what we want to know, that is, the questions to which an answer is needed. Moreover, the structure of a theoretical system tells us what alternatives are open in the possible answers to a given question (Parsons 1968, S. 9).

Die Zerlegung von Theorien in Frage-Antwort-Dyaden erlaubt mithin auf eine einfache und durchaus ,technische“ Weise die kontrollierte Abstraktion der Theorieinterpretation durch Abstraktion der Fragestellung (d.h. des Gesichtspunkts, unter dem die Theorie interessiert).

Diese Gesichtspunkte können in unterschiedlicher Abstraktionslage festgemacht werden. Webers Handlungstypologie in den „Grundbegriffen“ (Weber 2010) kann mit zunehmendem Abstraktionsgrad beispielsweise erstens als Antwort auf die Frage verstanden werden, unter welchen Gesichtspunkten menschliches Handeln rational ist; zweitens als Antwort auf die Frage, wie das wissenschaftliche Verstehen von Handlungssinn möglich ist; und drittens als Antwort auf die Frage verstanden werden, in welcher Sprache die Soziologie ihren Gegenstandsbereich beschreiben sollte. Je abstrakter die Frage gewählt wird, desto mehr mögliche Alternativen kommen in den Blick und desto mehr Theorien werden unter demselben Gesichtspunkt ver-

\footnotetext{
3 Das Frage/Antwort-Schema ist strukturverwandt, aber allgemeiner als das Problem/Lösungs-Schema. Die Behauptung, dass das Bild, das wir im Museum betrachten, ein Problem löst, geht nicht besonders leicht von der Zunge; wohl aber lässt sich (mit Gadamer) sagen, dass es eine Antwort auf eine im Kunstwerk selbst enthaltene Frage bietet. Das Problem/Lösungs-Schema ist daher besonders geeignet, um den Spezialfall des wissenschaftlichen Verstehens auf den Begriff zu bringen.
} 
gleichbar. Und da die problembezogene Interpretation von Theorien den Sinn für alternative Möglichkeiten schärft, ist das Problem/Lösungs-Schema nicht nur für die Bereitstellung von „Relationswissen“ (Greshoff 1999, S. 16) über soziologische Theorien, sondern auch für die Aufdeckung neuer Spielräume, d.h. für Möglichkeiten zu kreativer Theoriearbeit geeignet. Das explizite Bewusstsein für die theoretischen Bezugsprobleme eröffnet die Möglichkeit, kontrolliert über die Angemessenheit einer Problemstellung zu reflektieren - man sucht nicht nur nach der besten Antwort auf eine vorgegebene Frage, sondern fragt darüber hinaus: „Müsste man diese Frage nicht anders stellen?" In den folgenden Studien wird die explizite Nutzung des Schemas in der Theoriekonstruktion durch einschlägige Autoren nachgewiesen, wobei auch die vielseitigen Einsatz- und Variationsmöglichkeiten des Schemas deutlich werden.

\section{Drei Studien - Bourdieu, Habermas, Luhmann}

Nicht nur die Selbstverständigung im Fach, auch die Praxis soziologischer Theorie erfordert die Verarbeitung sachlicher Komplexität durch Theorienvergleiche. Der Anspruch auf die Neuheit einer Theorie setzt mindestens implizit einen Theorienvergleich voraus. Neuheit impliziert Differenz. Außerdem reagiert die Theoriekonstruktion häufig auf Probleme, die sich erst mit der Hilfe anderer Theorien wahrnehmen lassen. Das Problem/Lösungs-Schema eignet sich daher nicht nur für die Beschreibung der Relation zwischen bereits ausgearbeiteten Theorien, sondern auch für die Anleitung des theorienvergleichenden Anteils der Praxis der Theoriekonstruktion.

Das theorienvergleichende Moment ist besonders während der „Eröffnungszüge“ (Abbott 2004) der Theoriekonstruktion von Belang. Zu Beginn werden grundlegende theoretische Weichen gestellt und die im Bestehen begriffene Theorie verortet sich im Raum soziologischer Theorie. Dies soll an Fallbeispielen aus der soziologischen Theoriegeschichte gezeigt werden, indem Techniken der Theoriekonstruktion aus den Texten kreativer, relativ allgemein anerkannter, aber in ihrem Stil unterschiedlicher Theoretiker herausgearbeitet werden. Bei Bourdieu, Habermas und Luhmann interessieren uns jene typischerweise zu Beginn der Theorieentwicklung verfassten Texte, in denen sie ihre eigenen Entwürfe von theoretischen Vorlagen inspirieren lassen und von konkurrierenden Theorien abheben. Bei Bourdieu analysieren wir den zuerst 1972 auf Französisch erschienenen Entwurf einer Theorie der Praxis (Bourdieu 2012). Bei Habermas behandeln wir den Text „Zum Theorienvergleich in der Soziologie am Beispiel der Theorie der sozialen Evolutionstheorie“" (Habermas 1995) und bei Luhmann den Zeitschriftenaufsatz „Talcott Parsons - Zur Zukunft eines Theorieprogramms“ (Luhmann 1980). Die folgende Rekonstruktion der Texte erhebt keinen Anspruch auf besondere Originalität oder außergewöhnliche Tiefe der inhaltlichen Interpretation; das Ziel ist lediglich, auf eine theorietechnische Konvergenz aufmerksam zu machen. Das konvergente technische Dreischritt-Schema der Theoriekonstruktion, das wir nachweisen wollen, ist äußerst einfach: Über die problembezogene Rekonstruktion von Theorien erarbeiten sich die Autoren ein bestimmtes Problemniveau (1); über die Kritik an den bestehenden theoretischen Problemlösungen schaffen sie Platz für die von ihnen favorisierte Alternative (2) 
und reklamieren eine überlegene Lösung für die (typischerweise leicht verschobene) Problemstellung (3).

\subsection{Bourdieus problembezogene Argumentation in Entwurf einer Theorie der Praxis}

Bourdieu beginnt seine Theorie der Praxis bekanntlich nicht mit theoretischen Erörterungen, sondern mit drei Studien zu kabylischer Ethnologie. Diese sind ihrer Thematik (Ehre, Geschlechterverhältnisse sowie Heiratsregeln) geeignet, in die theoretische Problemstellung einzuführen, nehmen diese aber nicht vorweg. Am konkreten Material wird das zentrale Problem immer wieder angespielt, in seiner theoretischen Relevanz jedoch erst im zweiten Teil voll entfaltet.

In diesem zweiten Teil lässt sich die Strategie der Theoriekonstruktion durch problembezogenen Theorienvergleich klar nachweisen. Das zentrale Thema und theoretische Bezugsproblem der Theorie der Praxis ist die Reproduktion und Änderung von sozialen Strukturen, also das Problem der Strukturierung. Speziell geht es um den Modus, in dem sich Strukturierung vollzieht. Als mögliche Lösungen des Problems unterscheidet Bourdieu zwei holzschnittartig präsentierte Optionen. Anthropologie oder Soziologie könnten entweder ,subjektivistisch“ oder ,phänomenologisch“ an der Teilnehmerperspektive orientiert oder „objektivistisch“ an den übersubjektiven Regeln orientiert sein, die die Reproduktion der Praxis aus Beobachtersicht möglich machen. Das Verhältnis dieser beiden Perspektiven wird anhand ihrer Bezugsprobleme beschrieben. Der Subjektivismus verlege sich auf die Darstellung von Erfahrungswelten in ihrer Natürlichkeit und sei daran interessiert, wie die Teilnehmer ihre Welt erfahren, während der Objektivismus mit dieser Natürlichkeit breche und ,die [Frage, F. A.] nach den (besonderen) Bedingungen der Möglichkeit dieser Erfahrung selbst“" stelle (Bourdieu 2012, S. 147). Die beiden Perspektiven werden als miteinander unvereinbar gedacht; die Wahl der einen schließe die andere aus. Der Strukturalismus ignoriere das Welterleben der Akteure, während der Subjektivismus die objektiven Voraussetzungen der Strukturreproduktion nicht in den Blick bekomme. Auf diese Weise konstruiert Bourdieu zwei theoretische Strömungen mit unterschiedlichen Bezugsproblemen, aber auch zwei „blinde Flecken“, bei denen das jeweils von der einen Richtung beleuchtete in der anderen dunkel bleibt.

Diese theoretische Ausgangssituation lässt sich grafisch im Schema von Problem und Lösung darstellen (Abb. 1). Bourdieu unterscheidet nicht nur subjektivistische $\left(T_{a}\right)$ und objektivistische Theorien $\left(T_{b}\right)$, sondern auch ihre Problemstellungen $\left(P_{a}\right.$; $\mathrm{P}_{\mathrm{b}}$ ); d.h. er benennt unterschiedliche Fragen, auf die sie jeweils eine Antwort bieten. Die Pfeile zwischen Lösung und Problem rekonstruieren weder die zeitliche Struktur des Prozesses der Theorieentwicklung noch den Verlauf der theoretischen Argumentation, sondern die logischen Beziehungen zwischen Theorien/Theoremen und ihren Bezugsproblemen. Die Pfeilrichtung läuft stets von konkret zu abstrakt. 
Abb. 1 Bourdieus Ausgangssituation

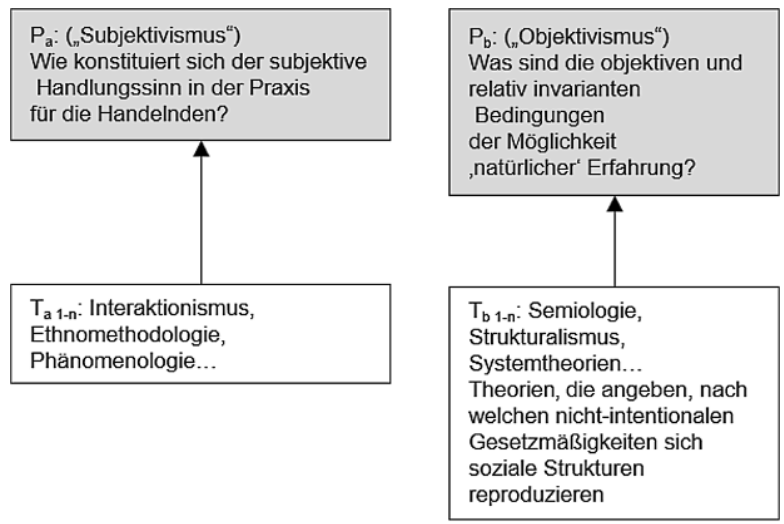

Hermeneutischer Bezug: spezifisch - abstrakt

Probleme sind abstrakter als Theorien, weil sie unterschiedliche Lösungen zulassen, Lösungen aber typischerweise auf bestimmte Probleme abstellen. ${ }^{4}$

Es kann kaum genug betont werden, wie wichtig diese Differenzierung von Problemstellung und Lösung für Bourdieus Prozess der Theoriekonstruktion ist. Jemand, der primär auf der Ebene von Theorien $(\mathrm{T})$ denkt, wird für Verbesserungen oder Weiterentwicklungen automatisch an der internen Struktur der Theorien ansetzen und versuchen, Unstimmigkeiten im Rahmen bestehender Problem- und Lösungskonstellationen zu beseitigen. Die naheliegende Art, die Theorie der Praxis in ihrem Verhältnis zu diesen Alternativen zu positionieren, bestünde nun darin, sie als einen Mittelweg zwischen Objektivismus und Subjektivismus zu präsentieren, der beide Lösungen vereint und sowohl die objektiven Regeln der Strukturreproduktion als auch den subjektiven Sinn des Handelns wissenschaftlich zugänglich macht. Die Integration liefe dann auf der Ebene der Lösungen, indem theoretische Ansätze, die bisher als inkommensurabel wahrgenommen wurden, in ihren Grundbegrifflichkeiten miteinander vermittelt werden, sodass beide Probleme gleichzeitig adressiert werden könnten. Diese Möglichkeit wird von Bourdieu jedoch bei genauer Lektüre in der Theorie der Praxis nicht gewählt. Der Subjektivismus ist für ihn keine gleichberechtigte Perspektive, sondern - ohne einen Bruch gegenüber der natürlichen Einstellung der Handelnden - bloß eine „Bestandsaufnahme des krud gegebenen“" (Bourdieu 2012, S. 150, kursiv i.O.). Der eigentliche Sinn subjektiv sinnvoller Handlungen erschließe sich erst aus ihrer objektiven Funktion in der Praxis.

\footnotetext{
4 Wenn das Problem ist, dass die Tür über den Boden schleift, kann man die Türbänder verstellen, das Türblatt kürzen, den Boden abschleifen oder einen Fitschenring in das Scharnier legen. Der Fitschenring hingegen erhöht die Position der Tür im Rahmen und tut sonst wenig Interessantes. Sprechakttheoretisch zeigt sich dieses Allgemeinheitsgefälle zwischen Frage und Antwort daran, dass Fragen Sprecher nicht auf die Richtigkeit eines propositionalen Gehalts verpflichten (,Ist es drei Uhr?“), während Antworten (,Es ist drei Uhr.") als Festlegung wirken und man sich beschweren kann, wenn die Auskunft nicht stimmt. „Abstraktion“ kann also über eine verminderte Festlegung auf bestimmte Inhalte charakterisiert werden.
} 
Die Theorieentwicklung läuft daher nicht über den Versuch, die beiden schon in sich heterogenen Strömungen zu vereinigen, sondern über eine Kritik des $O b$ jektivismus. Kritik meint sowohl den versuchten Nachweis einer unzureichenden Problemlösung des Objektivismus als auch eine Untersuchung der Voraussetzungen, die objektivistische Urteile ,allererst“ (Kant) möglich machen.

Er stellt also die Frage nach der Frage, die der objektivistischen Theorie zugrunde liegt:

Wie die objektivistische Erfahrung die Frage nach den Bedingungen der Möglichkeit primärer Erfahrung stellt und darin aufdeckt, wie diese sich grundlegend durch das Fehlen einer derartigen Frage definiert, so stellt wiederum die praxeologische Erkenntnis die objektivistische auf ihre Füße, indem sie nach den - theoretischen und gesellschaftlichen - Bedingungen der Möglichkeit auch dieser Frage selbst fragt und an den Tag bringt, daß sich die objektivistische Erkenntnisweise gleichermaßen und grundlegend durch den Ausschluß dieser Frage definiert (Bourdieu 2012, S. 148, Hervorhebung F. A.).

Diese Theoriestrategie ist auf den ersten Blick kontraintuitiv. Bourdieu beansprucht, dass die Reflexion auf die Grundlagen der bereits hochabstrakten objektivistischen Ansätze (er behandelt vor allem die Semiologie von Lévi-Strauss und ihre Grundlage im Strukturalismus von de Saussure) näher an den eigentlichen Sinn konkreter Handlungspraxen heranführt. Dies ist nur möglich, weil die Grundlagenreflexion nicht epistemologisch, sondern wissenssoziologisch durchgeführt wird: Zentrale Bedingung der Möglichkeit objektivistischer Betrachtungen ist für Bourdieu nicht ihre Erkenntnistheorie, sondern das tatsächliche, von Handlungsdruck entlastete Gelehrtendasein. Der Objektivismus neige zu einer mechanistischen oder deterministischen Handlungstheorie, weil das eigene Interesse an der Reproduktion von Bedeutungen und an der Interpretation von Sinn unreflektiert auf die handelnden Akteure übertragen werde (vgl. Bourdieu 2012, S. 141 f.; 158ff.) - die Logik der strukturalen Modelle werde mit der Logik der Praxis verwechselt (Lizardo 2010, S. 667). Um der Alternative von Subjektivismus und Objektivismus zu entgehen, sei es erforderlich, dass ,man sich über die Produktions- und Funktionsweise der praktischen Beherrschung verständigt, die eine objektiv intelligible Praxis sowie eine objektiv verzauberte Erfahrung dieser Praxis ermöglicht ..." (Bourdieu 2012, S. 149). Diese rhetorisch als Integrationsformel präsentierte Formulierung enthält die objektivistische Frage nach den Bedingungen der Möglichkeit einer sinnhaften Praxis, ist aber kein Angebot zur Übernahme der Problemstellung des „Subjektivismus“ nach der Konstitution der sinnhaften Erfahrung im Handeln (die als vorwissenschaftlich verworfen wird). Aus der Sicht der Bourdieuschen Theorie der Praxis sind die Erfahrungen und Zeugnisse der an der Praxis Beteiligten zwar wichtige Aspekte die ,Verzauberung“ ist motivstiftend und insofern handlungsrelevant -, aber der eigentliche Sinn der Handlungen erschließt sich erst aus der objektiven Funktion, die Handlungen und Verhaltensweisen als implizite Strategien in der Praxis einnehmen.

Die Problemstellung des Subjektivismus wird also erheblich modifiziert und auf die Analyse des praxisnotwendigen Mischverhältnisses von Anerkennung und Verkennung der Praxis durch die Akteure bezogen. Diese sowohl gegenüber dem Subjektivismus als auch gegenüber dem Objektivismus gleichermaßen ideologiekriti- 
sche Figur ermöglicht es Bourdieu, den Objektivismus gewissermaßen zu unterwandern, seine Problemstellung der tendenziellen Reproduktion sozialer Strukturen zu übernehmen, aber diese Reproduktion nicht von den Strukturen, sondern von dem kollektiven Habitus als „Erzeugungsbedingungen“ (Bourdieu 2012, S. 168) der Praxis aus zu denken. Die durch den „Subjektivismus“ in den Mittelpunkt gestellten Akteursperspektiven sind demgegenüber nachgeordnet, insofern sich ihr soziologischer Sinn erst aus der Kenntnis der objektiven Strukturen erschließt. Ob man in der Bourdieuschen Theorie der Praxis also einen Mittelweg zwischen Subjektivismus und Objektivismus oder eher eine Spielart des Objektivismus sehen will, hängt davon $\mathrm{ab}$, ob man die phänomenologische Problemstellung des Subjektivismus in der Problemverschiebung durch Bourdieu noch wiedererkennt. Derartige Klassifikationsfragen brauchen uns an dieser Stelle jedoch nicht zu interessieren (und die Unschärfe von Bourdieus Gegensatzpaar von Objektivismus und Subjektivismus und insbesondere die Diversität der als subjektivistisch etikettierten Ansätze stünden einer erhellenden Diskussion ohnehin im Wege). Es geht uns allein um die Technik der vergleichend-kontrastiven Theorieentwicklung in der Theorie der Praxis. Welche Theorietechnik zeigt sich in dieser Argumentation und inwiefern lässt sie sich im Vokabular des Theorienvergleichs rekonstruieren?

Zunächst ist festzustellen, dass Bourdieu nicht an Annahmen von Theorien (Lösungen), sondern an ihren Problemstellungen ansetzt. Bourdieu fragt nach der Frage hinter der Frage und transzendiert dadurch die etablierte Problemstellung des Objektivismus. Erst durch diese Problemverschiebung kann eine neue Lösung erarbeitet und im wissenschaftlichen Diskurs positioniert werden: die Theorie der Praxis.

Diese zweifellos äußerst erfolgreiche Strategie lässt sich in Abb. 2 gebündelt darstellen. Von der theoretischen Ausgangssituation ausgehend (siehe oben, hier umrahmt) beginnt Bourdieus eigene Theoriekonstruktion mit der Frage nach der Bedingung der Möglichkeit des Objektivismus (Abb. 2, oben rechts). Die Frage beantwortet Bourdieu mit dem bereits erwähnten Theorem (t) der Distanz der intellektualistischen Weltanschauung zur Praxis, das wiederum die Prämisse für eine Verschiebung der Problemstellung des Objektivismus $\left(\mathrm{P}_{\mathrm{b}}{ }^{\prime}\right)$ ist: Wie kann objektive Strukturreproduktion erklärt werden, ohne den praktischen Sinn des Handelns außer Acht zu lassen? Diese verschobene Fragestellung ist bereits das Bezugsproblem für Bourdieus Praxistheorie. Von den Grundannahmen der Theorie der Praxis aus wird es auch möglich, Teile der Problemstellung des Subjektivismus zu berücksichtigen $\left(\mathrm{P}_{\mathrm{a}}{ }^{\prime}\right)$. Die Problemverschiebungen werden mithilfe einer gestrichelten Linie symbolisiert, die Ähnlichkeit zwischen ursprünglicher Problemstellung in der Ausgangssituation und verschobener Problemstellung zeigt. Die grafische Darstellung veranschaulicht also erstens die Theoriestruktur als Problem/Lösungs-Konstellation, zweitens zeigt sie die Kontinuität von Problemstellungen und deren Relevanz für die Theoriekonstruktion und drittens ist sie eine bündige Zusammenfassung von Bourdieus Überlegenheitsanspruch - das, was zuvor in zwei heterogenen Theorietraditionen nebeneinander stand, wird nun im Rahmen einer integrativen Theorie vermittelt. 


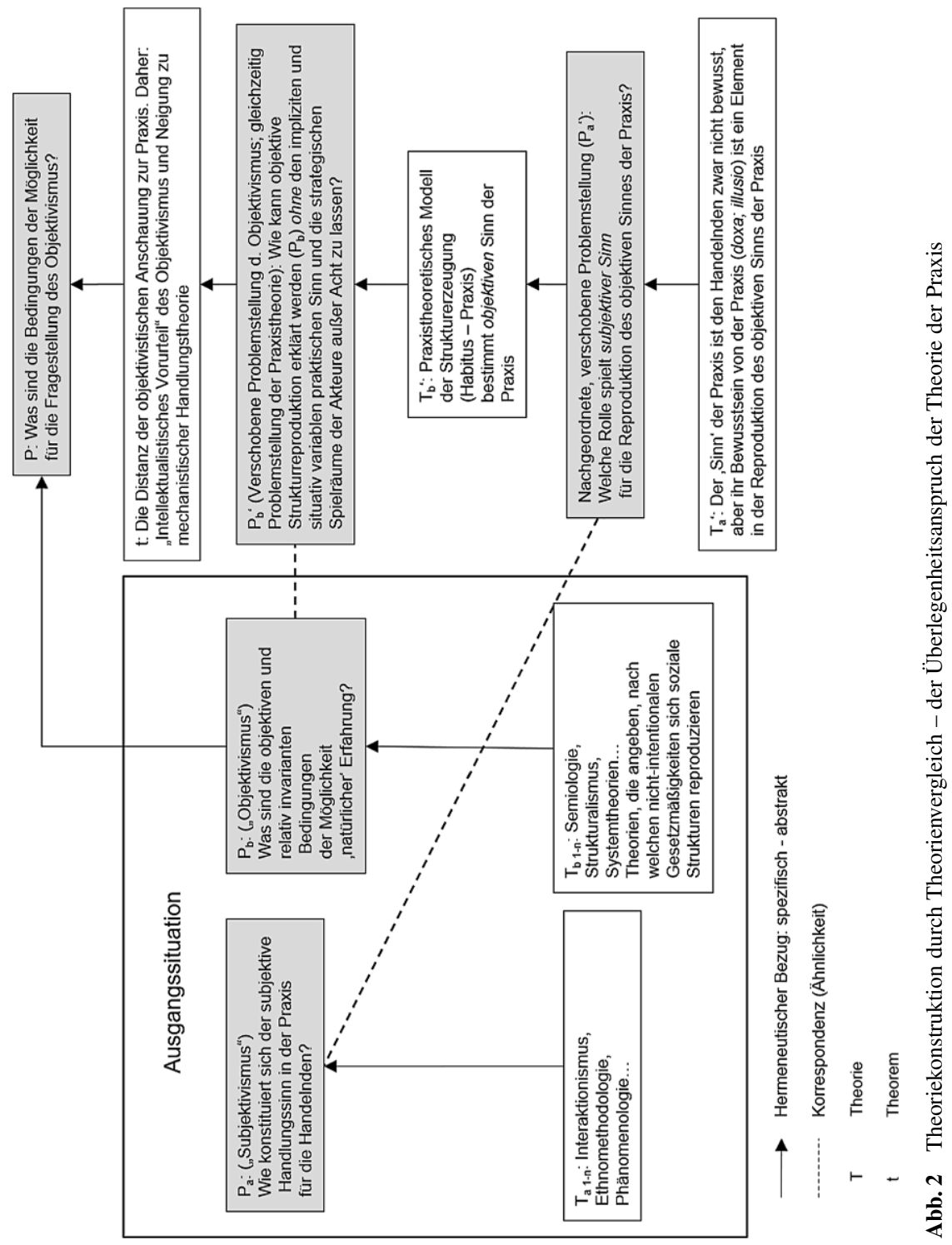

\subsection{Habermas' Rekonstruktion des historischen Materialismus}

Habermas weiß in außergewöhnlich hohem Maße die hermeneutische Auseinandersetzung mit theoretischen Traditionen für eigene produktive Theoriearbeit zu nutzen. In der Theorie des kommunikativen Handelns (TkH) findet er dafür die Formel der „Theoriegeschichte in systematischer Absicht“ (1981a, S. 201). Die Wahl eines Textes für unsere Zwecke ist daher äußerst willkürlich; nicht nur zu Beginn, sondern in fast jeder Veröffentlichung von Habermas ließe sich der problemzentrierte Umgang 
Abb. 3 Habermas' Ausgangssituation

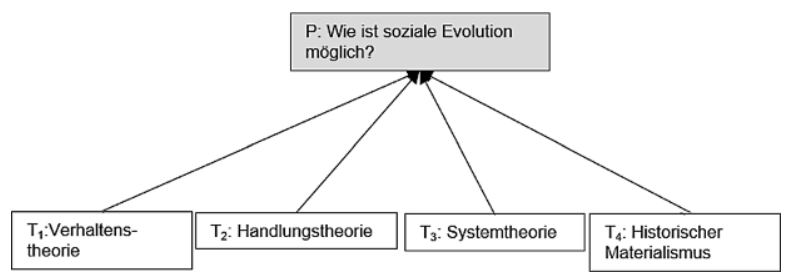

mit verschiedenen wissenschaftlichen Traditionen und ihre Transzendierung durch Kritik, Problemverschiebung und theoretische Innovation nachweisen. Wir wählen einen Text mit einer vergleichsweise einfachen Argumentation, in dem Habermas nicht nur theorienvergleichend vorgeht, sondern sein Vorgehen auch explizit als Theorienvergleich beschreibt. Es handelt sich um ein später im Band Zur Rekonstruktion des historischen Materialismus veröffentlichtes Manuskript mit dem Titel „Zum Theorienvergleich in der Soziologie: am Beispiel der Theorie der sozialen Evolutionstheorie“, das als Teil eines gemeinsamen Vortrags mit Klaus Eder beim Kasseler Soziologentag im Jahr 1974 referiert wurde (Habermas 1995). Das Thema des Vortrags war über die Rahmung des Soziologentages gesetzt, nämlich die Erklärung sozialer Evolutionsprozesse. Zu demselben Thema sprachen Niklas Luhmann, Karl-Dieter Opp und Karl Hermann Tjaden, deren systemtheoretische, verhaltenstheoretische sowie marxistische Erklärungsalternativen Anlass zur Abgrenzung boten. Wie leicht zu zeigen ist, entwickelt Habermas in dem Vortrag die gesellschaftstheoretische Problemstellung der zum Vortragszeitpunkt noch längst nicht in Aussicht stehenden Theorie des kommunikativen Handelns in einer im Vokabular von Problemstellung und -lösung gehaltenen Durcharbeitung durch theoretische Alternativen.

Er beginnt seinen Vortrag mit der Behauptung, dass noch keine adäquate Theorie der sozialen Evolution vorliege (Habermas 1995, S. 129). Daher nutzt er seinen Beitrag für einen programmatischen Entwurf, der seine Problemstellung gerade aus den Defiziten der bestehenden Ansätze gewinnt. Er geht in dem Vortrag (wohl auch beeinflusst von der Zusammensetzung des Kasseler Podiums) von vier Ansätzen aus:

1. Historischer Materialismus (Tjaden)

2. Handlungstheorie

3. Verhaltenstheorie (Behaviorismus) (Opp)

4. Funktionalistische Systemtheorie (Luhmann)

Auch diese Ausgangssituation lässt sich grafisch darstellen (Abb. 3). Habermas wird diese Konstellation der Theoriekonkurrenz gegen Ende seines Vortrags für einen kritisch-abgrenzenden Theorienvergleich nutzen, auf dessen Inhalt und methodologische Grundlagen hier nicht im Detail eingegangen werden soll (zur Funktion von Theorienvergleichen für Theoriekonkurrenz s. Anicker 2017), dessen Kriterien aber einiges über Habermas' eigenes Verständnis der theoretischen Funktion des Problem/Lösungs-Schemas für die Theoriekonstruktion verraten. Er kritisiert an den anderen in Kassel vorgestellten Ansätzen entweder, dass sie bestimmte Probleme a) nicht stellen (,Verhaltenstheorie“/Opp), b) nicht lösen (,Marxismus“/ 
Tjaden) oder c) den Zusammenhang von Problem/Lösung nicht theoretisch begründen können (,Systemtheorie“/Luhmann). Das Habermas'sche Theorieverständnis drückt sich insbesondere in seinem Vorwurf an die Adresse Luhmanns aus. Dessen Theorie sei „überabstrakt“, sodass sich „,die Wahl von Bezugsproblemen nicht mehr theoretisch rechtfertigen“ lasse (Habermas 1995, S. 141). Aus Habermas' Sicht ist es also eine der Aufgaben der Grundlagentheorie, mithilfe ihres sozialtheoretischen Vokabulars ihre Bezugsprobleme so zu koordinieren und zu spezifizieren, dass Wahlfreiheiten durch bestimmte Problemvorgaben eingeschränkt werden. Theoriebildung erscheint als Prozess der sukzessiven Bindung theoretischer Willkürfreiheit durch Verpflichtung auf immer spezifischere Problemstellungen und -lösungen.

Dies passt dazu, dass der theoriekonstruktive Teil seines Theorienvergleichs auf die Spezifikation einer Problemstellung hinausläuft. Habermas trifft seine erste eigene theoretische Festlegung, indem er sich die Problemstellung des historischen Materialismus zu eigen macht. Das heißt er legt den Begriff der „Erklärung“ sozialer Evolution so aus, dass die explanativen Ansprüche einer historisch-materialistischen Erklärung erfüllt sein müssen:

Eine Theorie der sozialen Evolution sollte als Gattungsgeschichte derart konzipiert werden, daß mindestens drei Probleme geklärt werden können: der Übergang zu Hochkulturen und damit die Entstehung von Klassengesellschaften; der Übergang zur Moderne und damit die Entstehung kapitalistischer Gesellschaften; schließlich die Dynamik einer antagonistischen Weltgesellschaft (Habermas 1995, S. 129-130).

Er ergänzt als weiteres Gütekriterium die Reflexivität (Selbstanwendbarkeit) der Theorie, so dass sie ,sowohl ihren eigenen Entstehungszusammenhang wie ihre möglichen Funktionen in gegebenen gesellschaftlichen Kontexten erklären kann“ (Habermas 1995, S. 130). Diese Affirmation der Problemstellung ist für Habermas mit einer deutlichen Ablehnung der Problemlösung des historischen Materialismus kompatibel:

Ich sehe andererseits nicht, warum diese Intentionen mich verpflichten sollten, die Konstruktionsmittel und die speziellen Annahmen einer im 19. Jahrhundert wurzelnden Theorie mehr oder weniger dogmatisch zu übernehmen ... (Habermas 1995, S. 130).

Habermas betreibt den historischen Materialismus, auf dessen Rekonstruktion er Anspruch erhebt, also über die Fortführung seiner Problemstellung unabhängig von seinen theoretischen Annahmen. Die Beibehaltung der Problemstellung verpflichtet nicht auf die Übernahme der sozialtheoretischen Grundlagen. Um diesen Spielraum zu nutzen und in ein neues sozialtheoretisches Grundvokabular einzuführen, behauptet Habermas, dass die Hauptdifferenz der kulturellen gegenüber der biologischen Evolution ihre Bindung an Kommunikation sei. Er fordert daher, dass die Evolutionstheorie in einem kommunikationstheoretischen und handlungstheoretischen Grundvokabular abgefasst sein sollte und weiterhin, dass Handlungstheorie systematisch als Kommunikationstheorie zu entwickeln sei. Im Durchgang durch die Grundbegriffe einer solchen Theorie (Sinn, pragmatische Universalien, Geltungsansprüche, Kommunikationsmedien usw.) zeigt sich, dass Habermas schon 1974 einen relativ 
klaren Begriff von der handlungstheoretischen Problemstellung der TkH hat. Theorietechnisch dient das übergeordnete Problem der adäquaten Erklärung von Evolutionsprozessen als „evolutionäres Lernen“ (Habermas 1995, S. 133) der Koordination der untergeordneten Problemstellungen der Handlungs- und Rationalitätstheorie, die zur Erklärung sozialer Evolutionsprozesse vonnöten sind.

Aus dem Horizont einer am Rationalitätsbegriff orientierten Handlungstheorie kann dann die Fragestellung der Evolutionstheorie nochmals genauer formuliert und der explanative Anspruch klarer spezifiziert werden. Die Forderungen an eine evolutionäre Erklärung sind (vgl. Habermas 1995, S. 134):

- analytisch trennscharfe Bestimmung von Entwicklungsdimensionen,

- Angabe von Lernmechanismen,

- Unterscheidung von Klassen von handlungsbezogenen Problemen (Habermas nennt technische Verfügungsprobleme versus konsensuelle Regelung von Handlungskonflikten),

- Differenzierung von Stufen der kognitiven, sprachlichen und interaktiven Entwicklung.

Habermas behauptet dann am Modellfall der moralischen Entwicklung, dass sich in diesen Dimensionen hierarchisch geordnete Strukturen nachweisen ließen, anhand derer sich bestimmte Zäsuren oder Stufen der Menschheitsentwicklung unterscheiden ließen; dass also der vorher zum Gegenstand der Evolutionstheorie erklärte Übergang zwischen vorhochkulturellen Gesellschaften, archaischen Hochkulturen, entwickelten Hochkulturen und früher Moderne handlungstheoretisch, d. h. durch eine den obigen Forderungen genügende Theorie, auszuweisen ist. Er behauptet, dass sich diese Stufen der gesellschaftlichen Entwicklung über den Modus der Konfliktregelung und unter anderem anhand der Trennung von kognitiver und normativer Rationalisierung unterscheiden lassen (vgl. Habermas 1995, S. 135).

Gerade weil der Text eine theoretische Lösung antizipiert, deren Publikation noch mehr als eine halbe Dekade entfernt ist, arbeitet Habermas besonders stark problemzentriert und die theorietechnische Trennung zwischen Problemstellung und -lösung tritt klar hervor. Das Ergebnis des Vortrags, seine „These“, ist denn auch keine Behauptung, sondern eine Auslegung der Fragestellung der Evolutionstheorie:

Ich möchte meine These dahingehend zusammenfassen, daß sich der evolutionäre Lernprozeß der Menschengattung im Rahmen einer Theorie begreifen läßt, die die evolutionären Errungenschaften von Gesellschaftssystemen durch eine Verknüpfung von zwei Fragestellungen erklärt: a) welche Steuerungsprobleme sind innovativ gelöst worden? b) durch welche Lernkompetenzen sind solche Innovationen möglich geworden? (Habermas 1995, S. 136, Hervorhebung F. A.).

Damit endet der theoriekonstruktive Teil des Vortrags. Die Theoriekonstruktion besteht hier also in der Ausarbeitung einer zweistufigen Problemstellung, die bereits die Konturen einer dualistisch konzipierten Evolutionstheorie der Gesellschaft erahnen lässt. Die Modifikation der Problemstellung des historischen Materialismus und ihre Neuformulierung auf Basis handlungstheoretischer Grundannahmen wird in Abb. 4 dargestellt. 


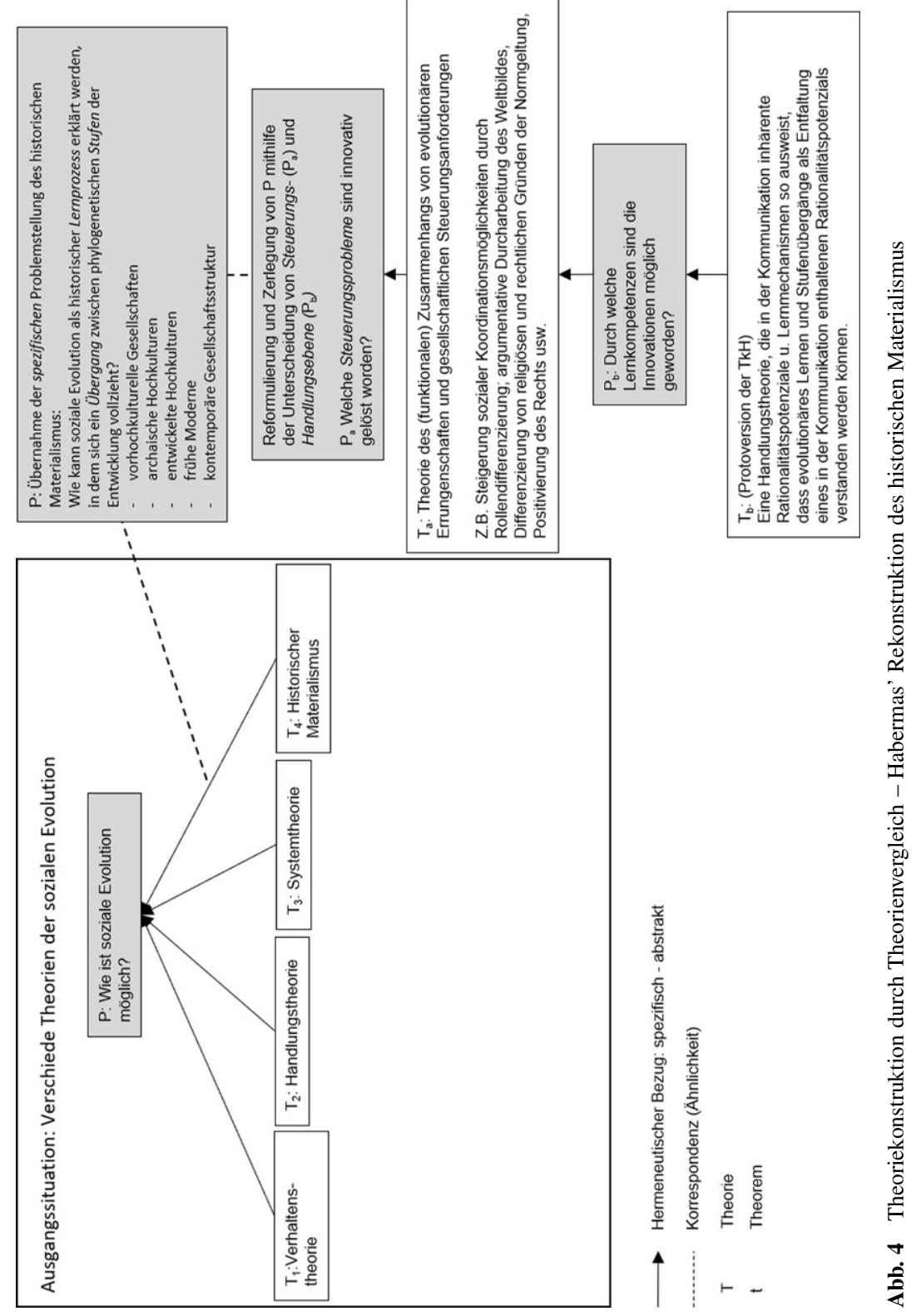




\subsection{Luhmanns Transzendierung der strukturfunktionalistischen Systemtheorie}

Niklas Luhmanns Theorieentwicklung zeichnet sich insbesondere zu Beginn durch großes in den Texten mitgeführtes methodisches Selbstbewusstsein aus. Theorieentscheidungen werden als Selektionen aus einem Horizont theoretischer Optionen kenntlich gemacht. Dabei bedient sich Luhmann besonders in frühen Texten explizit und in reflektierter Weise des Schemas von Problem/Lösung. Die Unterscheidung von Problemen und Lösungen dient in Gestalt der funktionalistischen Methode sowohl der Komplexitätsbewältigung durch Theorien (Luhmann 2005b, 2005c) als auch der Komplexitätsbewältigung der Gesellschaft selbst, die ihre Probleme durch Bildung sozialer Strukturen löst. In der frühen Phase seiner Theorieentwicklung trennt Luhmann allerdings noch stärker theorietechnische von empirischen Problemen, die lediglich im notwendig diffusen „Dachproblem“ der Reduktion von Komplexität zusammengeführt werden können (vgl. Luhmann 2005a, S. 328). ${ }^{5}$

Problemstellungen haben für Luhmann die größtmögliche Relevanz, sie sind, wenn man von Vergessen absieht, die einzige Möglichkeit des Umgangs mit wissenschaftlicher Komplexität:

Probleme ordnen die sich durchhaltende Kontinuität der abendländischen Denkgeschichte, die sich als einen einheitlichen Zusammenhang ausweist, in dem Antworten und deren Folgeprobleme wechseln können. Die Artikulation von Problemen ist Voraussetzung dafür, daß Alternativen entworfen und gegeneinander abgewogen werden können; ... Daraus muß man folgern, daß Probleme den Identitätskern einer Wissenschaft bilden, mit dessen Hilfe sie Theorien wechseln kann. Das legt es nahe, den Theorien selbst die Form einer Auslegung der Probleme auf mögliche Antworten hin zu geben (Luhmann 2005a, S. 326).

Die kognitive Strukturierungsleistung, die Probleme für die Wissenschaft insgesamt erbringen, dient Luhmann als Argument dafür, das Problem/Lösungs-Schema auch für die interne Organisation von Theorien $\mathrm{zu}$ verwenden, sodass die Systemtheorie als intern strukturierte Abfolge von Problemstellungen und -lösungen unterschiedlichen Abstraktionsgrads analysiert werden kann.

Typisch für Luhmann ist es, fremde Theorien vor allem über ihre Problemstellungen und kaum über ihre materialen Annahmen zu rezipieren (etwa, wenn Klassiker als Vertreter von Theorieentwürfen verstanden werden, deren Lösungen veraltet, deren Problemstellungen aber noch von Interesse seien, vgl. Luhmann 1988). Häufig findet sich folgendes Muster: Eine theoretische Tradition wird erwähnt, es wird geprüft, inwiefern bestimmte Aspekte ihrer Problemstellung in der Systemtheorie einen Platz finden können und falls dies der Fall ist, wird beiläufig angemerkt, dass diesbezügliche Anfragen nun an eine neue Adresse zu richten seien. Dies hat

\footnotetext{
5 Die früh gesehene Identität von Theorie- und Gegenstandsproblematik auf höchster Abstraktionsebene führt bekanntlich in späteren Entwürfen dazu, dass die Semantik der theoretischen Selbstbeschreibung und das Theorievokabular der Systemtheorie immer mehr zusammenfließen, sodass schließlich die Beobachtung zur Grundoperation und Paradoxieentfaltung das empirisch zu prozessierende Problem der Systeme selbst werden kann.
} 
einen abstrahierenden und eigentümlich distanzierenden Effekt, der nicht nur von textästhetischer Relevanz ist (Luhmann scheint eher über dem Theoriediskurs zu schweben, als an ihm teilzunehmen), sondern auch sachlich den Zweck hat, Anschlusschancen durch größtmögliche Abstraktion zu maximieren. Nach Auskunft von Dirk Bäcker interessierte sich Luhmann beim Lesen von Theorien fast ausschließlich für Problemstellungen und gelungene Formulierungen (Vorwort zu Luhmann 2005d). Eine Ausnahme besteht allerdings in der explizit als Vorgängerin gewürdigten Parsons'schen Systemtheorie. Wir diskutieren exemplarisch den Zeitschriftenaufsatz „Talcott Parsons - Zur Zukunft eines Theorieprogramms“ (Luhmann 1980). Wie im Folgenden zu zeigen ist, rekonstruiert Luhmann die Theorie zwar problembezogen, lässt sich aber zunächst auf ihre theoretische Eigenlogik ein. Die sich dabei zeigenden offenen Fragen und Probleme nutzt er als Möglichkeit, um alternative theoretische Optionen - eine neue Art von Systemtheorie - zu erschließen.

Für Luhmann entscheidet sich an der Art des Anschlusses an Parsons, ob Theorien von vergleichbarer „Reichweite und Anspruchslage“ im Fach weiter durchführbar sind und mithin das der Soziologie mögliche Theorieniveau (Luhmann 1980, S. 5). Die implizit mitlaufende Grundfrage der Untersuchung ist die nach der adäquaten Form einer fachuniversellen Theorie. Parsons' Theorie behandelt er auf dem größtmöglichen Abstraktionsniveau, d.h. auf der Ebene ihres obersten Bezugsproblems und den Grundentscheidungen zu seiner Lösung. Er möchte nicht die theoretischen Annahmen, sondern die Entscheidungen und Optionen beleuchten, auf denen sie beruhen (vgl. Luhmann 1980, S. 5). Seiner Auslegung nach basiert die Theorie auf zwei Eröffnungszügen: erstens der Analyse des Handlungsbegriffs über seine notwendigen Bestandteile und zweitens auf der Interpretation des Resultats als System - „Jede Handlung ist schon als Relation ihrer Komponenten System ...“ (Luhmann 1980, S. 8).

Luhmann nimmt nun nicht die Entfaltung dieses Programms, sondern die Begründbarkeit dieser Theorieentscheidung in den Blick: „Warum wird so prozediert?“ (Luhmann 1980, S. 6). Er greift insbesondere zwei theorieinterne Probleme heraus: erstens die Klärung der Emergenz von Handlungen und zweitens den Zusammenhang von Handlungs- und Systemtheorie. Bezüglich des ersten Problems diskutiert er die beiden theoretischen Alternativen, die Einheit der Handlung entweder aus der Relation ihrer Komponenten oder aus ihrem Zusammenhang mit anderen Handlungen abzuleiten. Die zweite Lösung mache es erforderlich, den bloß ,,analytischen“ Begriff des Systems fallen zu lassen, da Handlungen von empirischen Handlungssystemen konstituiert werden. Bezüglich der ersten Lösung müsse geklärt werden, wie die analytischen Elemente einer Handlung in der Handlung zur Handlungseinheit zusammengeschlossen werden. Die relationierende Relation sei zunächst die Selektion der Handlung durch normative Vorerwartungen, die Akteur und Situation zusammenbindet. Luhmann orientiert sich nicht an einzelnen Aussagen Parsons', sondern an der Relation von Problemstellung und dem Funktionsganzen der Theorie. In Hinblick auf die Theorie sei es z.B. ,[t]rotz entgegenstehender Äußerungen des Autors“ (Luhmann 1980, S. 8), konsistenter, den Systemcharakter von Handlungen bereits aus der Relation ihrer Komponenten abzuleiten, statt aus ihrer Relation zu anderen Handlungen. 
Abb. 5 Problembezogene Rekonstruktion von Parsons' Theorie durch Luhmann

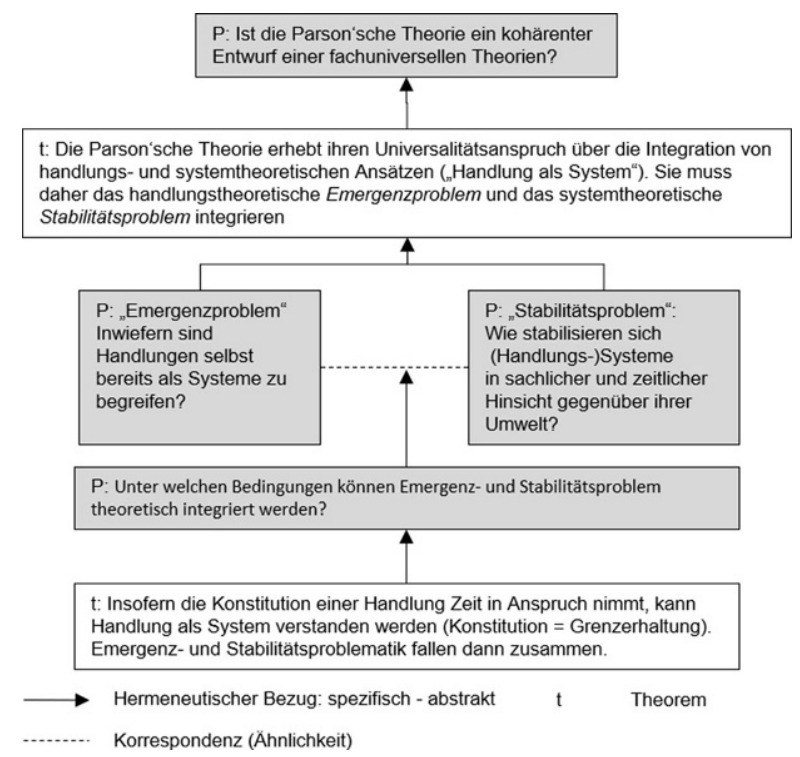

Das zweite Problem, das Luhmann herausgreift, ist die Vermittlung zwischen handlungs- und systemtheoretischen Problemstellungen in der Theorie selbst. Luhmann vertritt die These, dass aus der Problemstellung der Handlungsanalyse - ,Wie emergiert eine symbolisch generalisierte Handlungseinheit?" - die ordnungstheoretische Problemstellung der Systemtheorie (bearbeitet über das AGIL-Schema) nicht abgeleitet werden kann. Das (handlungstheoretische) Emergenzproblem - d.h. die gewissermaßen , interne“ Emergenz der Handlungseinheit - und das (systemtheoretische) Stabilitätsproblem, d.h. die ,externe“ Stabilität einer Handlungseinheit in der Zeit und gegenüber ihrer Umwelt seien anders gelagert und bei Parsons gebe es keine befriedigende Klärung ihres Zusammenhangs (vgl. Luhmann 1980, S. 9). Auf grundbegrifflicher Ebene sieht Luhmann allerdings die Möglichkeit, die beiden Problemstellungen in ein Verhältnis wechselseitiger Bedingtheit zu bringen:

Die Antwort kann aber lauten: daß die Einheit einer Handlung, da sie über selektive Prozesse konstituiert werden muß, Zeit in Anspruch nehmen und sich gegen Ausgesondertes oder Irrelevantes abgrenzen muß. Die Emergenz von Handlung ist, in anderen Worten, die Emergenz von Systemproblemen (Luhmann 1980, S. 10).

Damit entwickelt Luhmann in wenigen hochabstrakten Anmerkungen eine Möglichkeit, die häufig diagnostizierte Kluft zwischen Handlungs- und Systemtheorie (z. B. Habermas 1981b) zu schließen (zusammengefasst in Abb. 5). Er meint aber, dass die gedankliche Einheit der Theoriegrundlagen von sekundärer Bedeutung gegenüber der Einheitlichkeit der Theoriedurchführung und Konkretisierung am Gegenstand sei. Hier polemisiert er mit der Behauptung, dass im Zuge der Ausarbeitung der Theorie ,die Einheit der Grundlagen durch die Einheit der Kreuztabelle ersetzt ${ }^{\text {“ }}$ würde (Luhmann 1980, S. 10, kursiv i. O.).Der methodische Zwang, das AGIL-Sche- 
ma in Form wiederholter Kreuztabellierung anzuwenden, führe dazu, dass grundbegriffliche Weiterentwicklungen ohne Relevanz für die empirische Leistungsfähigkeit der Theorie blieben (vgl. Luhmann 1980, S. 10f.).

Nach dieser weitgehend internen Rekonstruktion verlässt Luhmann die Binnenperspektive der Parsons'schen Systemtheorie. Er geht von Problemen, die sich aus dem Horizont der Theorie selbst stellen, zu allgemeinen fachlichen Problemen über. Insbesondere seien es zwei relevante Problemstellungen, die mithilfe der Parsons'schen Systemtheorie nicht adäquat bearbeitet werden könnten: „das Problem der Selbstreferenz“ und „das Problem der Komplexität“ (Luhmann 1980, S. 12). Bezüglich des ersten Problems vermutet Luhmann, dass sich erst über den Begriff der Selbstreferenz kybernetische Steuerungshierarchien und damit die Selbstregulation des Umweltkontaktes adäquat beschreiben lassen. Nur wenn es der Theorie gelänge, über die Randvariablen des AGIL-Schemas (d.h. System/Umwelt und die Zeitdimension, die in der instrumental-konsumatorischen Dimension enthalten ist) sich selbst als System zu begreifen, d. h. in sich selbst vorzukommen, könne an neuere Forschungsinteressen angeschlossen werden. Luhmann sieht dafür zwar Ansätze in der Theorie, nicht jedoch ihre systematische Ausarbeitung. Durch die Kreuztabellentechnik könne Selbstreferenz lediglich als Differenzierungsfolge (nicht: ihre Bedingung) und über den Gebrauch von Austauschmedien gedacht werden, wobei Luhmann skeptisch ist, ob diese theoretische Platzierung der Bedeutung der kybernetischen Systemtheorie gerecht werden kann (Luhmann 1980, S. 14).

Das Problem der Komplexität sei vor allem in den wissenschaftstheoretischen Voraussetzungen des ,,analytischen Realismus“ (Parsons' erkenntnistheoretische Position) berücksichtigt. Der analytische Realismus reflektiere die bewusst in die Theorie aufgenommene Notwendigkeit selektiver Komplexitätsreduktion. Allerdings werde der Stellenwert des Komplexitätsproblems nicht ausreichend berücksichtigt. Die Theorie verschließe sich beispielsweise den Einsichten, dass Rationalität sich nur über den Umgang mit Komplexität begreifen lasse und dass Komplexitätsreduktion nicht nur in der Theorie, sondern auch im Gegenstandsbereich der Soziologie von größter Bedeutung sei. Komplexität müsse ,,als ein nichthintergehbares Universalphänomen“ verstanden werden, das ,nicht nur im Verhältnis von Erkenntnis und Gegenstand ein Problem ist, sondern den Aufbau (die ,Selbstorganisation“) der Realität selbst bedingt““ (Luhmann 1980, S. 14). ${ }^{6}$ Letztendlich ließen sich damit methodologische und ordnungstheoretische Fragen - das „Hobbesian problem of order" - auf dasselbe Grundproblem, die Reduktion von Komplexität, zurückbeziehen. Diese Letztbegründung der Einheit von Theorie und Gegenstand im Problem der Komplexität eröffnet für Luhmann sowohl die Möglichkeit, die Parsons'sche Theorie zu integrieren und an neuere Entwicklungen (also vor allem Luhmanns eigene Theorieinteressen) anzuschließen, aber auch über alternative Problemlösungen nachzudenken. In der Schlusspassage lässt er am Horizont seiner Rekonstruktionsversuche die Möglichkeit einer anderen Systemtheorie auftauchen, die Selbstrefe-

\footnotetext{
6 Auffällig ist an dieser Stelle natürlich der theoriestrategische Kniff, mit dem Luhmann den analytischen Realismus auf Basis einer Unterscheidung kritisiert, die er selbst nicht treffen würde - die zwischen der Funktionslogik theoretischer und realer Systeme. Letztere wäre für Parsons ja immer nur durch erstere zugänglich.
} 


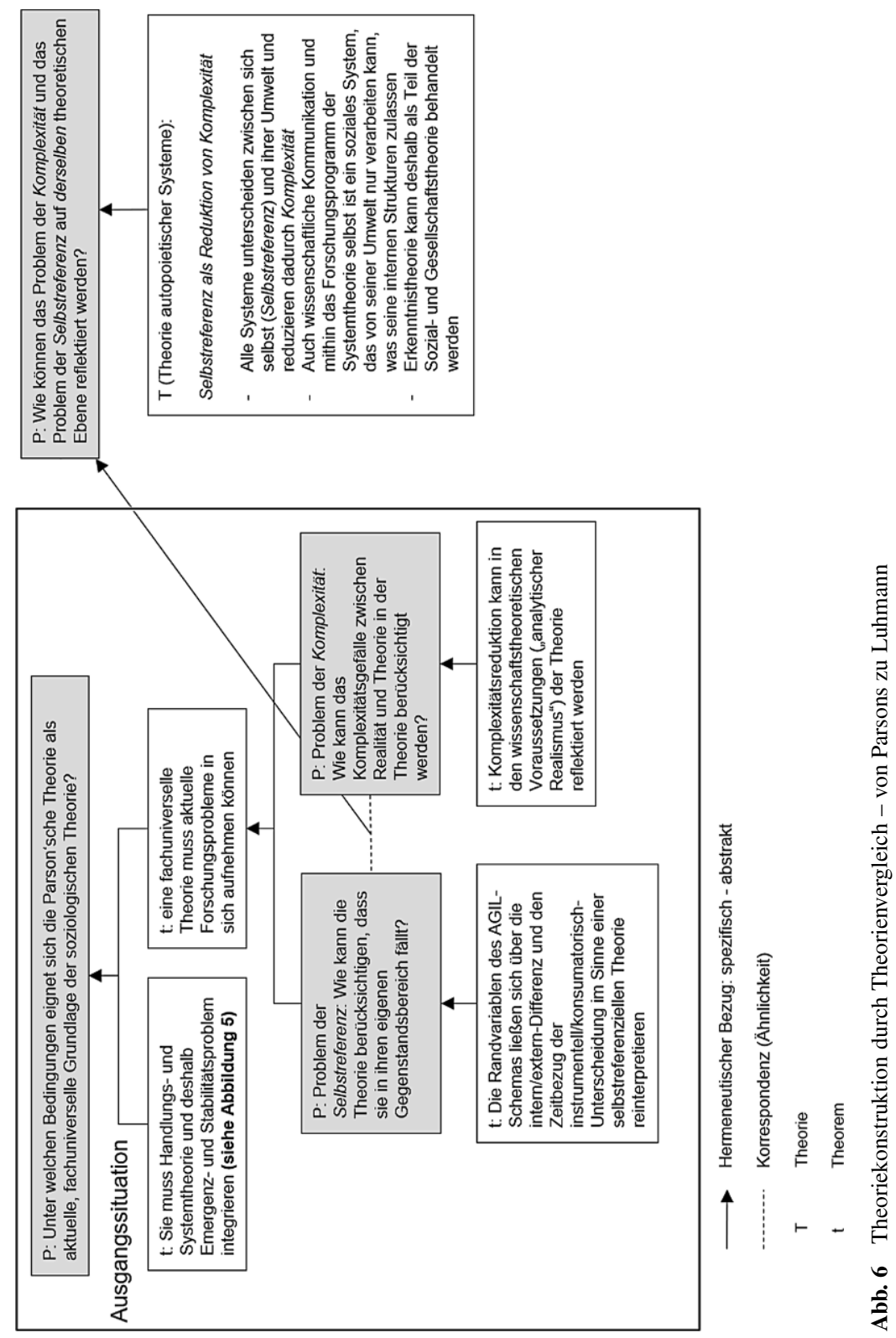

renz und Komplexität nicht indirekt reflektiert, sondern zu ihrem Ausgangspunkt macht (s. Abb. 6). Wegen ihrer rhetorischen Brillanz sei diese Schlussbemerkung hier in voller Länge zitiert:

Diese Rückübersetzung [Luhmanns Rekonstruktion, F. A.] vermittelt den Anschluß an neuere Entwicklungen der kybernetischen Systemtheorie. Sie bringt ein Einheitsmotiv der allgemeinen Theorie des Handlungssystems zu Tage, das 
ihr selbst unzugänglich geblieben ist. Sie klärt grundlegende Optionen dieser Theorie und versetzt sie zurück in den Zustand einer Frage. Diese Frage lautet: Ist die Selektivität, zu der alle Komplexität zwingt, zureichend erfaßt, wenn man sie auf der Ebene der Erkenntnistheorie bzw. Methodologie als „Analytik" und wenn man sie auf der Ebene der Handlung als normative Orientierung bzw. als Wert begreift? Stellt man sich einmal diese Frage, dann ist der Eindruck nicht mehr abzuweisen, daß das Theorieprogramm von Parsons seine Einheit erst an einem Punkt gewinnt, der zugleich die Möglichkeit gibt, über Alternativen nachzudenken (Luhmann 1980, S. 15f.).

Luhmann überlässt es seinen Lesern, aus der Kritik an der Theorie die im Text immer wieder angespielte Alternative einer anderen Systemtheorie zu folgern. Der bekannte dramaturgische Kunstgriff des offenen Schlusses involviert die Aufmerksamkeit und lenkt sie in eine bestimmte Richtung, die wegen der Genrezugehörigkeit des Textes jedoch auf der Hand liegt: Statt die Probleme der Selbstreferenz und der Komplexitätsreduktion mithilfe disparater Bestandteile der Parsons'schen Systemtheorie zu thematisieren, sollte die Systemtheorie selbst als Auslegung dieser Probleme und also als eine Theorie selbstreferenzieller und komplexitätsreduzierender Systeme angelegt werden. Alle Systeme reduzieren Komplexität, indem sie sich selbst von ihrer Umwelt unterscheiden und nur jene Umweltaspekte verarbeiten, die an die eigenen Systemstrukturen anschlussfähig sind. Diese These macht es möglich, für die Probleme von Komplexitätsreduktion und Selbstreferenz dieselbe theoretische Lösung zu postulieren, denn die Komplexitätsreduktion sozialer Systeme läuft über ihre Selbstreferenz. Die Probleme von Selbstreferenz und Komplexität haben als bei allen Analysen mitlaufende Problematik (es kann immer gefragt werden, auf welchen internen Strukturen die Außenwahrnehmung von Systemen beruht, was dabei ausgeblendet wird und welche anderen Komplexitätsreduktionen möglich gewesen wären) eine wesentlich höhere Signifikanz als im Fall der Parsons'schen Theorie. Insofern also Selbstreferenz als die Grundbedingung der Stabilisierung aller sozialen Systeme betrachtet wird, ist die komplexitätsreduzierende Selektivität der Systemtheorie selbst (welche Parsons im Rahmen des analytischen Realismus reflektiert) ein wichtiger Spezialfall der Selektivität aller System-Umwelt-Verhältnisse. Diese Problematik der Selbstreferenz im engeren Sinne reflektiert die Theorie, indem sie sich selbst unter ihren Gegenständen wiederentdeckt. Sie ist ein „Teilsystem (Soziologie) eines Teilsystems (Wissenschaft), des Gesellschaftssystems“ (Luhmann 1984, S. 34) und kann sich durch den Vergleich anderer sozialer Systeme mit dem System systemtheoretischer Kommunikation über sich selbst aufklären.

\section{Ein allgemeines Schema: Theoriekonstruktion durch Theorienvergleich}

Unsere Studien zeigen, dass das methodische Selbstverständnis von Bourdieu, Habermas und Luhmann sich offensichtlich am Problem/Lösungs-Schema orientiert, dass sie dieses nutzen, um verschiedene Theorien auf geteilte Probleme zu beziehen und so vergleichbar zu machen und dass sich ihre schrittweise Transzendierung der 
von ihnen selbst beschriebenen theoretischen Ausgangssituation als Problem- und Lösungsverschiebung rekonstruieren lässt. Ihre Auseinandersetzung mit der theoretischen Tradition ist deshalb nie bloß ritualisierte Verneigung vor vergangenen Geistesgrößen, sondern selbst ein produktiver Akt.

Die behandelten Autoren erschließen durch Vorgängertheorien jene zunächst theoriespezifischen Problemstellungen, die wegen ihres höheren Abstraktionsgrades immer auch andere theoretische Optionen eröffnen. In „Bewegung“ gerät die Auslegung durch Kritik an Problemstellung oder -lösung. Sie markieren Schwierigkeiten und Defizite bei der Lösung des Problems, die den Wechsel zu einer anderen Theorie motivieren und gleichzeitig der Entwicklung dieser Theorie eine Richtung geben können. Kontinuität zum bestehenden theoretischen Diskurs wird über die Beibehaltung von (modifizierten) Problemstellungen hergestellt, während sich Divergenz aus neuen theoretischen Annahmen ergibt. So kann theoretische Innovation gleichzeitig angeregt, kontrolliert und theorienvergleichend ausgewiesen werden. Der technische Aspekt dieser allgemeinen Strategie theoretischer Kreativität lässt sich nochmals stärker synthetisieren und kann analytisch in folgende Schritte zergliedert werden:

1. Problembezogene Interpretation: Eine oder mehrere Theorien werden als Auslegung einer ihr eigenen Problemstellung rekonstruiert. Nicht selten wird das Bezugsproblem der Theorie nicht vollständig aus ihr selbst entnommen, sondern relativ rasch verschoben (Luhmann, Bourdieu) oder ergänzt (Habermas).

2. Kritik: Entweder die Problemstellung oder der Zusammenhang zwischen Problem und Lösung werden kritisiert. Kritisiert man den Zusammenhang von Problem und Lösung, betreibt man zunächst ,interne“ Theoriekritik - etwa Luhmann, der zunächst diskutiert, inwiefern die Parsons'sche Synthese von Handlungs- und Systemtheorie mit ihren eigenen begrifflichen Mitteln als konsistent ausgewiesen werden kann, oder Habermas, der den theoretischen Anspruch des historischen Materialismus übernimmt, aber dessen theoretische Grundlagen aufgrund interner Schwierigkeiten ablehnt. Kritisiert man die Problemstellung, kann man entweder nach den theoretischen Voraussetzungen des Problems fragen und dadurch die Aufnahme von nichtreflektierten Voraussetzungen in die Theorie selbst anmahnen (hier Bourdieu, der nach den Bedingungen der Möglichkeit des „Objektivismus“ fragt), oder verlangen, dass weitere, nicht in der Theorie, wohl aber dem Fach selbst relevante Problemstellungen berücksichtigt werden (z. B. Luhmann, der die Möglichkeiten der Parsons'schen Theorie prüft, auf die Probleme von Selbstreferenz und Komplexität zu reagieren oder Habermas, der Reflexivität als Zusatzkriterium einführt).

3. Neuansatz: Schließlich wird ein Theorievorschlag gemacht, der vorsieht, entweder die Lösung oder Problem und Lösung zu wechseln. Im ersten Fall präsentiert sich die theoretische Innovation als bessere Alternative für die Lösung eines bekannten Problems (Bourdieus Theorie der Praxis als eine gegenüber dem Strukturalismus bessere Lösung des Problems der Strukturreproduktion). Im zweiten Fall wird zwar beides gewechselt, Kontinuität zur Ausgangstheorie und ihren Erkenntnisinteressen, wird aber über die kontrollierte Verschiebung der Problemstellung beibehalten; die Transformation der Problemstellung muss entweder aus den internen Theorieproblemen der Ausgangstheorie selbst oder aus allgemeinen wissen- 
schaftlichen Zielen begründet werden. Dieser Begründungsprozess verbindet neu und alt. Die neue Problemstellung ist also gewissermaßen eine nahe Verwandte der alten, die durch größere Eleganz und andere angenehme Eigenschaften besticht.

Der Nachweis dieser theorietechnischen Konvergenz hat einen doppelten Sinn: Weil gezeigt werden kann, dass die untersuchten Autoren, trotz starker Divergenz in Theorieverständnis und Art der Bezugnahme auf andere Theorien, dieselbe Technik der Theoriekonstruktion durch Theorienvergleich nutzen, ist ihre Sachadäquanz nahegelegt. Es mag andere Lösungen für das methodische Problem geben, wie Theorieentwürfe gleichzeitig Anspruch auf Neuheit und theoretische Überlegenheit erheben und dennoch an den etablierten Fachdiskurs angeschlossen werden können. Aber dass stilistisch sonst sehr verschiedene Theoretiker sich allesamt des Dreischritt-Schemas der überlegenen Problemlösung bedienen, lässt vermuten, dass es sich um eine relativ basale, diesseits der Verzweigung von Paradigmen angesiedelte Technik handelt und die Zahl der Alternativen begrenzt ist. Zudem wird durch den Nachweis der Konvergenz das theorietechnische Schema von den Kontexten seines Gebrauchs abstrahiert, sodass es möglich wird, eine allgemeine und von bestimmten „Schulen“ oder „Lagern“ unabhängige Technik der Theoriekonstruktion herauszuarbeiten. Die kämpferisch-polemische Inszenierung der Theorie der Praxis bedient sich ebenso dieser Theorietechnik wie die abstrakt-analytische Selbsttranszendierung der Systemtheorie oder die Überwindung und Fortführung des historischen Materialismus durch die Theorie des kommunikativen Handelns. Wenn dies richtig ist, steht der soziologischen Theorie eine von partikularen Theorien weitgehend unabhängige Technik des Theorizing zur Verfügung, die relativ leicht didaktisch vermittelbar ist und daher den Einstieg in die aktive Teilnahme am Fachdiskurs soziologischer Theorie erleichtern sollte. Da es sich gleichzeitig um eine Technik der Darstellung theoretischer Innovationen handelt, dient sie außerdem der Transparenz des Theoriediskurses selbst. Verkürzte oder einseitig verzerrte Vergleiche fallen viel stärker als solche ins Auge, wenn ein vollständiges Schema des Vergleichs vorliegt.

Selbstverständlich verbürgt die Technik der Theoriekonstruktion nicht die Richtigkeit ihrer Ergebnisse. Interpretationen der Ausgangstheorien können verzerrt, Problemstellungen irreführend und Lösungen unzutreffend sein. Das Ergebnis von Theorieprüfung und -kritik im Fachdiskurs kann nicht in der Phase der Theorieentwicklung vorweggenommen werden. Auch kann die Theorietechnik Theoriekompetenz und theoretische Kreativität zwar unterstützen, nicht aber ersetzen.

Bei aller notwendigen Absage an überzogene Hoffnungen sind jedoch zwei absehbare Einwände zurückzuweisen. Erstens wäre es falsch, in der theorienvergleichenden Theoriekonstruktion nur eine Technik der Theoriedarstellung zu sehen, die mit der eigentlichen theoretischen Entdeckung nichts zu tun hat. Die Technik eignet sich zwar gleichzeitig als legitime Form der Theorieinszenierung, aber ohne den bestehenden Theorie- und Problemkontext wäre die Neuheit generierende Verschiebung wohl kaum möglich gewesen. Inwiefern die Technik auch nach der Etablierung eines theoretischen Ansatzes genutzt wird, um beispielsweise Theorieintegration zu betreiben oder ob sie lediglich für den Take-off der Theorie - für ihre Abstoßbewegung, in der sie sich gleichzeitig auf die Tradition stützt und von ihr löst - relevant ist, ist eine Frage des Stils. Während Habermas ein ums andere Mal nachzuwei- 
sen bestrebt ist, dass sich der Begriff des kommunikativen Handelns als natürlicher Fluchtpunkt einer Vielzahl theoretischer Problemstellungen und ihrer klassischen Bearbeitung ergibt, verlässt sich Luhmann eher auf die Entfaltung einmal gefundener Ausgangsprobleme und begrifflicher Grundentscheidungen, während Bourdieu weitergehende theoretische Entwicklungen als Resultat empirischer Lernprozesse inszeniert.

Zweitens impliziert eine Theoriekonstruktion mittels Theorienvergleich keineswegs automatisch ein ,empiriefernes“ Theoriendesign, wie in der Theorizing-Diskussion oft unterstellt wird. Richtig ist zwar, dass sich über Theorienvergleiche Abstraktionsgewinne erzielen lassen, aber Abstraktion steht nicht im Gegensatz zu empirischer Fruchtbarkeit. Im Gegenteil ermöglicht Abstraktion zunächst eine durchaus wünschenswerte Ausweitung des empirischen Anwendungsbereichs von Theorien. ${ }^{7}$ Ausschlaggebend ist dann allerdings, ob die abstrakten Grundlagen einer Theorie für die Interpretation und Erklärung empirischer Sachverhalte fruchtbar gemacht werden können. Dies entscheidet sich vor allem an der Frage, ob sie auf kontrollierte Weise mit Methoden empirischer Forschung vermittelt werden können - etwa indem sie überprüfbare Hypothesen zu formulieren erlauben (Popper 1935, S 67 ff.; vgl. auch Schmid 2010) oder als Heuristiken der Gegenstandserschließung dienen (z. B. Lindemann 2008).

Des Weiteren ist die mangelnde empirische Anwendbarkeit von Theorien häufig ein wichtiges Kriterium der Theoriekritik (Schritt 2 in unserem Schema) und kann daher Anlass dafür bieten, Bezugsprobleme und theoretische Grundannahmen so weiterzuentwickeln, dass die empirische Aussagekraft von Theorien erhöht wird (vgl. etwa Tutić 2015; Anicker 2020). Das Problem des Bezugs theoretischer Aussagesysteme auf methodisch kontrollierte Erfahrung ist offenkundig genau das: ein Problem, auf dessen Lösung hin Theorien verglichen werden können. Gerade die Entwicklung von Theorien, die sich gegenüber konkurrierenden Angeboten durch größeren empirischen Gehalt auszeichnen, erfordert Abstraktion und Vergleich Kuscheln mit den Phänomenen reicht nicht.

Unsere Untersuchung bekräftigt mithin eine zunehmend den Charakter des Selbstverständlichen abstreifende Trivialität: Theoriebildung hat nicht nur im Vorfeld oder als Zusatzoption, sondern wesentlich etwas mit dem Lesen von anderen Theorien zu tun. Theorieinnovation bedarf der Auseinandersetzung mit der Tradition und wird erst dadurch zu einem Beitrag, der nicht den Narzissmus seines Urhebers, sondern die Erkenntnisinteressen des Fachs bedient. Dies ist kein Plädoyer für Konventionalismus, sondern für die Verbindung von Innovativität und Systematizität: Man

\footnotetext{
7 Je abstrakter theoretische Einheiten definiert werden, desto mehr empirische Phänomene können mithilfe einer Theorie erfasst werden und an umso mehr Phänomenen kann sich die Theorie potenziell bewähren - eine allgemeine Organisationssoziologie hat einen größeren empirischen Anwendungsbereich als eine reine Schul-, Verwaltungs- oder Betriebssoziologie; eine allgemeine Theorie der Gravitation ist empirisch gehaltvoller als eine Theorie, die nur die Schwerkraft auf der Erde erklärt. Abstrakte Theorien müssen freilich für empirische Erklärungen spezifiziert und in Zusammenhang mit Mess- oder Interpretationstheorien gebracht werden, was sicherlich nicht immer zufriedenstellend gelingt - aber dies rechtfertigt es keineswegs, das Spezielle pauschal gegen das Allgemeine auszuspielen. Nicht möglichst konkrete, faktennah angesiedelte Theorien sind wünschenswert, sondern Theorien, die eine nur durch abstrakte Grundannahmen erzielbare hohe Reichweite mit hoher empirischer Irritationsfähigkeit verbinden.
} 
kann echte soziologische Entdeckungen machen und zu theoretischen Innovationen kommen, indem man andere Theorien liest. Im Vorfeld der Theoriebildung ist daher der Kauf einer komfortablen Sitzgelegenheit anzuraten - vorzugsweise mit einer Möglichkeit, die oberen Extremitäten abzustützen.

Danksagung Für hilfreiche Kommentare und Diskussionen zu diesem Manuskript bedanke ich mich herzlich bei Joachim Renn, Peter Isenböck, Hartmut Esser, André Armbruster sowie den Herausgebern und den anonymen Gutachtern dieser Zeitschrift.

Funding Open Access funding enabled and organized by Projekt DEAL.

Open Access Dieser Artikel wird unter der Creative Commons Namensnennung 4.0 International Lizenz veröffentlicht, welche die Nutzung, Vervielfältigung, Bearbeitung, Verbreitung und Wiedergabe in jeglichem Medium und Format erlaubt, sofern Sie den/die ursprünglichen Autor(en) und die Quelle ordnungsgemäß nennen, einen Link zur Creative Commons Lizenz beifügen und angeben, ob Änderungen vorgenommen wurden.

Die in diesem Artikel enthaltenen Bilder und sonstiges Drittmaterial unterliegen ebenfalls der genannten Creative Commons Lizenz, sofern sich aus der Abbildungslegende nichts anderes ergibt. Sofern das betreffende Material nicht unter der genannten Creative Commons Lizenz steht und die betreffende Handlung nicht nach gesetzlichen Vorschriften erlaubt ist, ist für die oben aufgeführten Weiterverwendungen des Materials die Einwilligung des jeweiligen Rechteinhabers einzuholen.

Weitere Details zur Lizenz entnehmen Sie bitte der Lizenzinformation auf http://creativecommons.org/ licenses/by/4.0/deed.de.

\section{Literatur}

Abbott, Andrew Delano. 2004. Methods of Discovery. Heuristics for the Social Sciences. New York: Norton.

Abbott, Andrew. 2010. On Writing the Social Process. Vortrag an der Universität Bielefeld. Bielefeld. http://wwwhomes.uni-bielefeld.de/bghs/dokumente/ABBOTTBIEL.pdf (Zugegriffen: 27. Okt. 2019).

Abend, Gabriel. 2008. The Meaning of “Theory”. Sociological Theory 26:173-199.

Anicker, Fabian. 2017. Theorienvergleich als methodologischer Standard der soziologischen Theorie. Zeitschrift für Soziologie 46:71-88.

Anicker, Fabian. 2019. Theorie aus der Froschperspektive: Zu Richard Swedbergs ,theorizing“. Zeitschrift für Theoretische Soziologie 8:282-288.

Anicker, Fabian. 2020. Grundzüge einer empirischen Pragmatik des kommunikativen Handelns. Zeitschrift für Soziologie 49(1):28-48.

Balog, Andreas, und Johann A. Schülein (Hrsg.). 2008. Soziologie, eine multiparadigmatische Wissenschaft. Erkenntnisnotwendigkeit oder Übergangsstadium? Wiesbaden: Springer VS.

Baumann, Marcel M., und Thorsten Bonacker. 2008. Für einen Theoriendialog ohne Entscheidungszwang. Nutzen und Grenzen eines fallbezogenen Theorienvergleichs aus Sicht der empirischen Konfliktforschung. In Sozialtheorien im Vergleich, Hrsg. Thorsten Bonacker, Rainer Greshoff und Uwe Schimank, 229-248. Wiesbaden: Springer VS.

Bonacker, Thorsten, Rainer Greshoff und Uwe Schimank (Hrsg.). 2008. Sozialtheorien im Vergleich. Der Nordirlandkonflikt als Anwendungsfall. Wiesbaden: VS Verlag.

Bourdieu, Pierre. 2012. Entwurf einer Theorie der Praxis auf der ethnologischen Grundlage der kabylischen Gesellschaft. 3. Aufl. Frankfurt a.M.: Suhrkamp.

Bourdieu, Pierre, Jean-Claude Chamboredon und Jean-Claude Passeron. 2011. Soziologie als Beruf. Wissenschaftstheoretische Voraussetzung soziologischer Erkenntnisse. Berlin: de Gruyter.

Burzan, Nicole. 2019. Über eine multiparadigmatische Soziologie. Soziologie 48:28-36.

Carleheden, Mikael. 2016. What Conception of the Theoretical Does "Theorizing" Presuppose? Comment On Richard Swedberg's "Before Theory Comes Theorizing Or How to Make Social Science More Interesting". The British Journal of Sociology 67:36-42. 
Carleheden, Mikael. 2019. How to Theorize? On the Changing Role and Meaning of Theory in the Social Sciences. In Contemporary Philosophy and Social Science. An Interdisciplinary Dialogue, Hrsg. Michiru Nagatsu und Attilia Ruzzene, 311-331. New York: Bloomsbury Academic.

Collins, Randall. 1998. The Sociological Eye and Its Blinders. Contemporary Sociology 27:2-7.

Esser, Hartmut. 1993. Soziologie. Allgemeine Grundlagen. Frankfurt: Campus.

Farzin, Sina, und Henning Laux (Hrsg.). 2014. Gründungsszenen soziologischer Theorie. Wiesbaden: Springer Fachmedien Wiesbaden.

Farzin, Sina, und Henning Laux. 2016. Gründungsszenen - Eröffnungszüge des Theoretisierens am Beispiel von Heinrich Popitz' Machtsoziologie. Zeitschrift für Soziologie 45:241-260.

Fleck, Ludwik. 1980. Entstehung und Entwicklung einer wissenschaftlichen Tatsache : Einführung in die Lehre vom Denkstil und Denkkollektiv. Frankfurt a.M.: Suhrkamp.

Gadamer, Hans-Georg. 1975. Wahrheit und Methode. 4. Aufl. Tübingen: J.C.B. Mohr.

Greshoff, Rainer. 1999. Lassen sich die Konzepte von Max Weber und Niklas Luhmann unter dem Aspekt „Struktur und Ereignis“ miteinander vermitteln? In Struktur und Ereignis in theorievergleichender Perspektive, Hrsg. Rainer Greshoff und Georg Kneer, 13-50. Opladen/Wiesbaden: Westdeutscher Verlag.

Greshoff, Rainer, und Uwe Schimank (Hrsg.). 2006. Integrative Sozialtheorie? Esser - Luhmann - Weber. Wiesbaden: VS Verlag.

Greshoff, Rainer, Gesa Lindemann und Uwe Schimank. 2007. Theorienvergleich und Theorienintegration - Disziplingeschichtliche und methodische Überlegungen zur Entwicklung eines paradigmenvermittelnden „,conceptual framework“ für die Soziologie. Diskussionspapier, Carl von Ossietzky Universität Oldenburg. https://www.uni-oldenburg.de/fileadmin/user_upload/sowi/ag/ast/download/dp/astdp-1-07.pdf. Zugegriffen: 27. Okt. 2019.

Habermas, Jürgen. 1981a. Theorie des kommunikativen Handelns, Bd. 1. Frankfurt a.M.: Suhrkamp.

Habermas, Jürgen. 1981b. Theorie des kommunikativen Handelns, Bd. 2. Frankfurt a.M.: Suhrkamp.

Habermas, Jürgen. 1995. Zum Theorienvergleich in der Soziologie: am Beispiel der Theorie der sozialen Evolutionstheorie. In Zur Rekonstruktion des historischen Materialismus, 6. Aufl., 129-143. Frankfurt a.M.: Suhrkamp.

Haller, Max. 1999. Soziologische Theorie im systematisch-kritischen Vergleich. Opladen: Leske + Budrich.

Hammond, Michael. 2018. "An Interesting Paper But Not Sufficiently Theoretical”: What Does Theorising In Social Research Look Like? Methodological Innovations 11:1-10.

Hondrich, Karl Otto. 1976. Entwicklungslinien und Möglichkeiten des Theorievergleichs. In Zwischenbilanz der Soziologie. Verhandlungen des 17. Deutschen Soziologentags, Hrsg. Rainer M. Lepsius. Stuttgart: Enke.

Kneer, Georg, und Markus Schroer. 2009. Soziologie als multiparadigmatische Wissenschaft. Eine Einleitung. In Handbuch Soziologische Theorien, Hrsg. Georg Kneer und Markus Schroer, 7-18: VS.

Krause, Monika. 2016. The Meanings of Theorizing. The British Journal of Sociology 67:23-29.

Kuhn, Thomas. 1970. The Structure of Scientific Revolutions. Chicago: University of Chicago Press.

Lakatos, Imre. 1982. Die Methodologie der wissenschaftlichen Forschungsprogramme. Braunschweig: Vieweg.

Lenger, Alexander, Tobias Rieder und Christian Schneickert. 2014. Theoriepräferenzen von Soziologiestudierenden. Welche Autor*innen Soziologiestudierende tatsächlich lesen. Soziologie 43:450-467.

Lindemann, Gesa. 2006. Die Emergenzfunktion und die konstitutive Funktion des Dritten: Perspektiven einer kritisch-systematischen Theorieentwicklung. Zeitschrift für Soziologie 35:82-101.

Lindemann, Gesa. 2008. Theoriekonstruktion und empirische Forschung. In Theoretische Empirie. Zur Relevanz qualitativer Forschung, Hrsg. Herbert Kalthoff, Stefan Hirschauer und Gesa Lindemann, 107-127. Frankfurt a.M.: Suhrkamp.

Lizardo, Omar. 2010. Beyond the Antinomies of Structure. Levi-Strauss, Giddens, Bourdieu, and Sewell. Theory and Society 39:651-688.

Luhmann, Niklas. 1980. Talcott Parsons - Zur Zukunft eines Theorieprogramms. Zeitschrift für Soziologie 9:5-17.

Luhmann, Niklas. 1984. Soziale Systeme. Frankfurt a. M.: Suhrkamp.

Luhmann, Niklas. 1988. Arbeitsteilung und Moral. Durkheims Theorie. In Emile Durkheim: Über soziale Arbeitsteilung. Studie über die Organisation höherer Gesellschaften, 2. Aufl., 19-38. Frankfurt a.M.: Suhrkamp.

Luhmann, Niklas. 1990. Die Wissenschaft der Gesellschaft. Frankfurt a.M.: Suhrkamp.

Luhmann, Niklas. 2005a. Die Praxis der Theorie. In Soziologische Aufklärung I. Aufsätze zur Theorie sozialer Systeme, 7. Aufl., 317-335. Wiesbaden: VS Verlag. 
Luhmann, Niklas. 2005b. Funktionale Methode und Systemtheorie. In Soziologische Aufklärung I. Aufsätze zur Theorie sozialer Systeme, 7. Aufl., 39-67. Wiesbaden: VS Verlag.

Luhmann, Niklas. 2005c. Funktion und Kausalität. In Soziologische Aufklärung I. Aufsätze zur Theorie sozialer Systeme, 7. Aufl., 11-38. Wiesbaden: VS Verlag.

Luhmann, Niklas. 2005d. Einführung in die Theorie der Gesellschaft. Hrsg. Dirk Bäcker, Heidelberg: CarlAuer.

Opp, Karl-Dieter, und Reinhard Wippler (Hrsg.). 1990. Empirischer Theorienvergleich. Erklärungen sozialen Verhaltens in Problemsituationen. Opladen: Westdeutscher Verlag.

Parsons, Talcott. 1968. The Structure of Social Action. a Study of Social Theory With Special Reference to a Group of Recent European Writers. Vol I: Marshall, Pareto, Durkheim. 4. Aufl., New York: Free Press.

Popper, Karl R. 1935. Logik der Forschung Zur Erkenntnistheorie der modernen Naturwissenschaft. Wien: Springer-Verlag

Popper, Karl R. 1952. The Nature of Philosophical Problems and Their Roots in Science. The British Journal for the Philosophy of Science 3:124-156.

Popper, Karl R. 1979. Ausgangspunkte. Meine intellektuelle Entwicklung. Hamburg: Hoffman \& Campe.

Reichenbach, Hans. 1983. Erfahrung und Prognose. Eine Analyse der Grundlagen und der Struktur der Erkenntnis. Wiesbaden: Springer Fachmedien.

Schmid, Michael. 2004. Rationales Handeln und soziale Prozesse. Beiträge zur soziologischen Theoriebildung. Wiesbaden: Springer VS.

Schmid, Michael. 2009. Theorien, Modelle und Erklärungen. Einige Grundprobleme des soziologischen Theorienvergleichs. In Neuer Mensch und kollektive Identität in der Kommunikationsgesellschaft, Hrsg. Preyer Gerhard, 323-359. Wiesbaden: VS Verlag für Sozialwissenschaften.

Schmid, Michael. 2010. Theorien mittlerer Reichweite. Versuch einer Problemklärung. Berliner Journal für Soziologie 20:383-400.

Schneider, Wolfgang Ludwig. 1991. Objektives Verstehen. Rekonstruktion eines Paradigmas. Gadamer, Popper, Toulmin, Luhmann. Opladen: Westdeutscher Verlag.

Schneider, Wolfgang Ludwig. 1999. Verschiedene Möglichkeiten Theorien miteinander zu vergleichen, oder: Wie vergleicht man Weber und Luhmann. In Struktur und Ereignis in theorievergleichender Perspektive. Ein diskursives Buchprojekt, Hrsg. Rainer Greshoff und Georg Kneer, 287-316. Opladen/Wiesbaden: Westdeutscher Verlag.

Schneider, Wolfgang Ludwig. 2008. Grundlagen der soziologischen Theorie. 3 Bände. 3. Aufl. Wiesbaden: Springer VS.

Swedberg, Richard. 2012. Theorizing in Sociology and Social Science: Turning to the Context of Discovery. Theory and Society 41:1-40.

Swedberg, Richard. 2014a. The Art of Social Theory. Princeton and London: Princeton University Press.

Swedberg, Richard (Hrsg.). 2014b. Theorizing In Social Science. the Context of Discovery. Stanford: Stanford Social Sciences.

Swedberg, Richard. 2017. Theorizing in Sociological Research: A New Perspective, a New Departure? Annual Review of Sociology 43:189-206.

Tavory, Iddo. 2016. The Pragmatist Wave of Theory Construction. The British Journal of Sociology 67:50-56.

Tomasello, Michael. 2006. Die kulturelle Entwicklung des menschlichen Denkens. Frankfurt a.M.: Suhrkamp.

Toulmin, Stephen E. 1983. Kritik der kollektiven Vernunft. Frankfurt a.M.: Suhrkamp.

Tutić, Andreas. 2015. Warum denn eigentlich nicht? Zur Axiomatisierung soziologischer Handlungstheorie. Zeitschrift für Soziologie 44:83-98.

Weber, Max. 2010. Wirtschaft und Gesellschaft. Grundriss der verstehenden Soziologie. Frankfurt am Main: Zweitausendeins.

Weick, Karl E. 1989. Theory Construction as Disciplined Imagination. Academy of Management Review 14:516-531.

Fabian Anicker 1986, Dr. phil., wissenschaftlicher Mitarbeiter und Redakteur der Zeitschrift für Theoretische Soziologie am Institut für Soziologie, WWU Münster. Forschungsgebiete: Soziologische Theorie; Politische Soziologie. Veröffentlichungen: Entwurf einer Soziologie der Deliberation. Weilerswist 2019; Grundzüge einer empirischen Pragmatik des kommunikativen Handelns. Zeitschrift für Soziologie 49, 2020; Theorienvergleich als methodologischer Standard der soziologischen Theorie. Zeitschrift für Soziologie 46, 2017. 